\title{
Cortical Pitch Regions in Humans Respond Primarily to Resolved Harmonics and Are Located in Specific Tonotopic Regions of Anterior Auditory Cortex
}

\author{
Sam Norman-Haignere, ${ }^{1,2}$ Nancy Kanwisher, ${ }^{1,2}$ and Josh H. McDermott ${ }^{2}$ \\ ${ }^{1} \mathrm{McGovern}$ Institute for Brain Research and ${ }^{2}$ Department of Brain and Cognitive Sciences, Massachusetts Institute of Technology, Cambridge, \\ Massachusetts 02139
}

Pitch is a defining perceptual property of many real-world sounds, including music and speech. Classically, theories of pitch perception have differentiated between temporal and spectral cues. These cues are rendered distinct by the frequency resolution of the ear, such that some frequencies produce "resolved" peaks of excitation in the cochlea, whereas others are "unresolved," providing a pitch cue only via their temporal fluctuations. Despite longstanding interest, the neural structures that process pitch, and their relationship to these cues, have remained controversial. Here, using fMRI in humans, we report the following: (1) consistent with previous reports, all subjects exhibited pitch-sensitive cortical regions that responded substantially more to harmonic tones than frequency-matched noise; (2) the response of these regions was mainly driven by spectrally resolved harmonics, although they also exhibited a weak but consistent response to unresolved harmonics relative to noise; (3) the response of pitch-sensitive regions to a parametric manipulation of resolvability tracked psychophysical discrimination thresholds for the same stimuli; and (4) pitch-sensitive regions were localized to specific tonotopic regions of anterior auditory cortex, extending from a low-frequency region of primary auditory cortex into a more anterior and less frequency-selective region of nonprimary auditory cortex. These results demonstrate that cortical pitch responses are located in a stereotyped region of anterior auditory cortex and are predominantly driven by resolved frequency components in a way that mirrors behavior.

Key words: pitch; auditory cortex; fMRI; tonotopy; resolved harmonics; periodicity

\section{Introduction}

Pitch is the perceptual correlate of periodicity (repetition in time), and is a fundamental component of human hearing (Plack et al., 2005). Many real-world sounds, including speech, music, animal vocalizations, and machine noises, are periodic, and are perceived as having a pitch corresponding to the repetition rate (the fundamental frequency or F0). Pitch is used to identify voices, to convey vocal emotion and musical structure, and to segregate and track sounds in auditory scenes. Here we investigate the cortical basis of pitch perception in humans.

When represented in the frequency domain, periodic sounds exhibit a characteristic pattern: power is concentrated at harmonics (multiples) of the fundamental frequency. Because the

\footnotetext{
Received July 5, 2013; revised 0ct. 23, 2013; accepted 0ct. 27, 2013.

Author contributions: S.V.N.-H., N.K., and J.M. designed research; S.V.N.-H. performed research; S.V.N.-H. analyzed data; S.V.N.-H., N.K., and J.M. wrote the paper.

This study was supported by the National Eye Institute (Grant EY13455 to N.K.) and the McDonnell Foundation (Scholar Award to J.M.). We thank Alain De Cheveigne, Daniel Pressnitzer, and Chris Plack for helpful discussions; Kerry Walker, Dan Bendor, and Marion Cousineau for comments on an earlier draft of this paper; and Jessica Pourian for assistance with data collection and analysis.

The authors declare no competing financial interests.

Correspondence should be addressed to Sam Norman-Haignere, MIT, 43 Vassar Street, 46-4141C, Cambridge, MA 02139. E-mail:svnh@mit.edu.

DOI:10.1523/JNEUROSCI.2880-13.2013

Copyright $\odot 2013$ the authors $\quad 0270-6474 / 13 / 3319451-19 \$ 15.00 / 0$
}

cochlea filters sounds into frequency bands of limited resolution, the frequency and time domain can in principle provide distinct information (Fig. 1). Models of pitch perception have thus focused on the relative importance of temporal versus spectral (frequency-based) pitch cues (Goldstein, 1973; Terhardt, 1974; Meddis and Hewitt, 1991; Patterson et al., 1992; Bernstein and Oxenham, 2005). A central finding in this debate is that sounds with perfect temporal periodicity, but with harmonics that are spaced too closely to be resolved by the cochlea, produce only a weak pitch percept (Houtsma and Smurzinski, 1990; Shackleton and Carlyon, 1994). This finding has been taken as evidence for the importance of spectral cues in pitch perception.

In contrast, the large majority of neuroimaging studies have focused on temporal pitch cues conveyed by unresolved pitch stimuli (Griffiths et al., 1998; Patterson et al., 2002; Hall et al., 2005; Barker et al., 2011). As a consequence, two questions remain unanswered. First, the relative contribution of spectral and temporal cues to cortical responses remains unclear. One previous study reported a region with greater responses to resolved than unresolved pitch stimuli (Penagos et al., 2004), but it is unknown whether this response preference is present throughout putative pitch regions and if it might underlie behavioral effects of resolvability. Second, the anatomical locus of pitch responses remains unclear. Some studies have reported pitch responses in a specific region near anterolateral Heschl's gyrus (HG) (Griffiths 
A Effect of Cochlear Filtering on Spectral Resolvability

Stimulus Spectrum

Simulated Excitation Pattern

$100 \mathrm{~Hz} F 0$

Harmonics 1 - 35
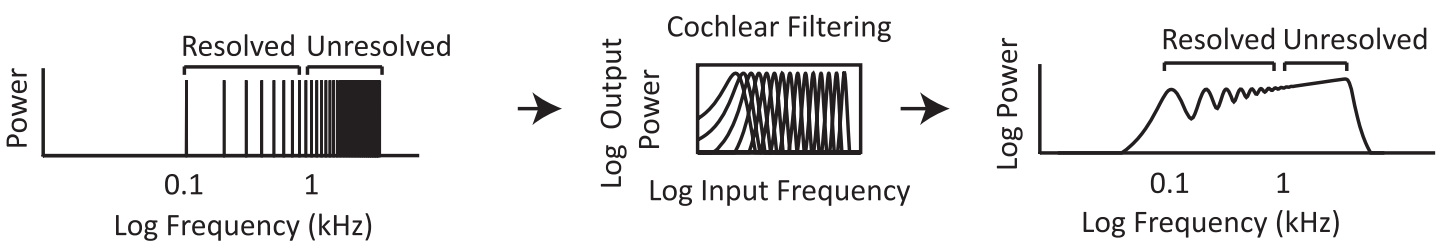

B Effect of Cochlear Filtering on Temporal Responses
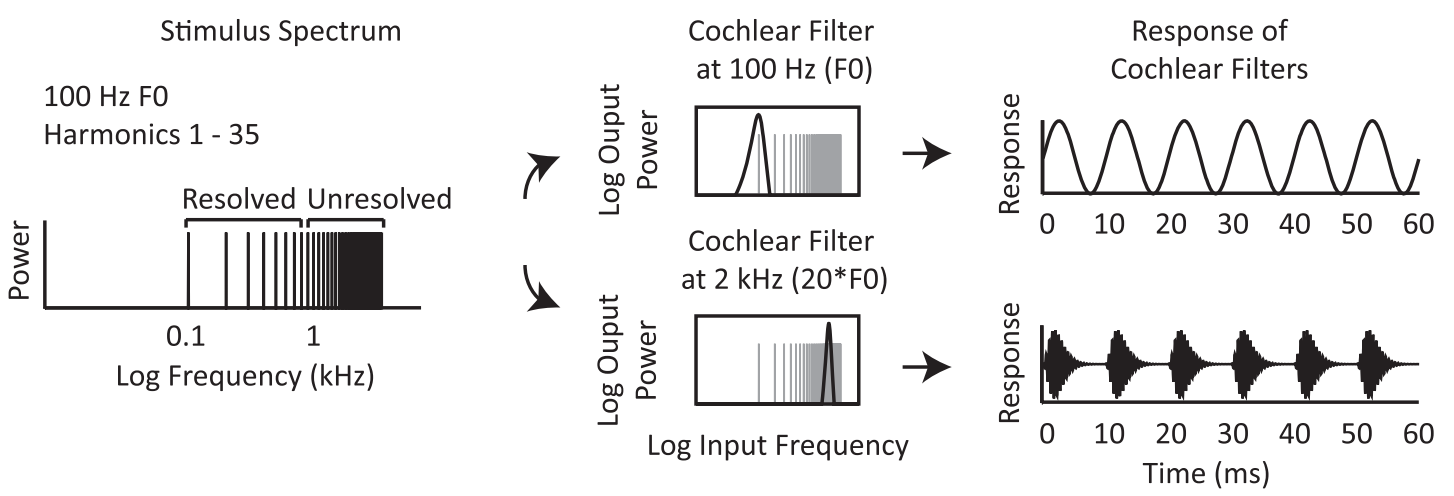

Figure 1. Effects of cochlear filtering on pitch cues. $A$, Spectral resolvability varies with harmonic number. Left, Power spectrum of an example periodic complex tone. Any periodic stimulus contains power at frequencies that are multiples of the $\mathrm{F} O$. These frequencies are equally spaced on a linear scale, but grow closer together on a logarithmic scale. Middle, Frequency response of a bank of gammatone filters (Slaney, 1998; Ellis, 2009) intended to mimic the frequency tuning of the cochlea. Cochlear filter bandwidths are relatively constant on a logarithmic frequency scale (though they do broaden at low frequencies). Right, Simulated excitation pattern (giving the magnitude of the cochlear response as a function of frequency) for the complex tone shown at left. Cochlear filtering has the effect of smoothing the original stimulus spectrum. Low-numbered harmonics yield resolvable peaks in the excitation pattern, providing a spectral cue to pitch. High-numbered harmonics, which are closely spaced on a log scale, are poorly resolved after filtering because many harmonics fall within a single filter and thus do not provide a spectral pitch cue. $\boldsymbol{B}$, Temporal pitch cues in the cochlea's output. Left, Power spectrum of a periodic complex tone. Middle, Frequency response of two example cochlear filters superimposed on the example stimulus spectrum. Right, Response of each example filter plotted over a brief temporal interval. Both resolved and unresolved harmonics produce periodic fluctuations in the temporal response of individual cochlear filters.

et al., 1998; Patterson et al., 2002), whereas other studies have reported relatively weak and distributed pitch responses (Hall and Plack, 2007, 2009). These inconsistencies could plausibly be due to the use of unresolved pitch stimuli: such stimuli produce a weak pitch percept, and might be expected to produce a correspondingly weak neural response.

Here we set out to answer these two questions. We first measured the response of pitch-sensitive regions to stimuli that parametrically varied in resolvability. These analyses revealed larger responses to resolved than unresolved harmonics throughout cortical pitch-sensitive regions, the parametric dependence of which on resolvability closely tracked a standard behavioral measure of pitch perception. We then used the robust cortical response to resolved harmonics to measure the anatomical and tonotopic location of pitch responses in individual subjects. In the Discussion, we show how our results help to reconcile a number of apparently divergent results from the prior literature.

\section{Materials and Methods}

Overview

Our study was composed of three parts. In Part 1, we measured the response of pitch-sensitive voxels in auditory cortex to a parametric manipulation of resolvability. We identified pitch-sensitive voxels based on a greater response to harmonic tones than noise, irrespective of resolvability, and then measured their response to resolved and unresolved stimuli in independent data. In Part 2, we measured the anatomical distribution of pitch-sensitive voxels across different regions of auditory cortex and tested whether any response preference for resolved harmonics is found throughout different cortical regions. In Part 3, we tested whether pitch responses occur in specific regions of the cortical tonotopic map. We examined tonotopy because it one of the most well established organizational principles in the auditory system and because neurophysiology studies have suggested that pitch-tuned neurons are localized to specific tonotopic regions of auditory cortex in the marmoset (Bendor and Wang, 2005, 2010), raising the question of whether a homologous organization is present in humans. We also conducted a follow-up experiment to rule out the possibility that our results can be indirectly explained by frequency adaptation. For clarity, this control experiment is described in a separate section at the end of the Methods and Results.

For the purposes of this paper, we refer to a greater response to harmonic tones than to frequency-matched noise a "pitch response" and we refer to brain regions or voxels with pitch responses as "pitch-sensitive." We use these terms for ease of discussion, acknowledging that the precise role of these regions in pitch perception remains to be determined, a topic that we return to in the Discussion. We begin with some background information on how harmonic resolvability can be manipulated experimentally.

\section{Background}

A large behavioral literature has converged on the idea that pitch perception in humans depends on the presence of harmonic frequency components that are individually resolved on the cochlea-that is, that produce detectable peaks in the cochlea's excitation pattern (Oxenham, 2012). 


\section{A Simulated Excitation Patterns for Example Notes from Different Stimulus Conditions}

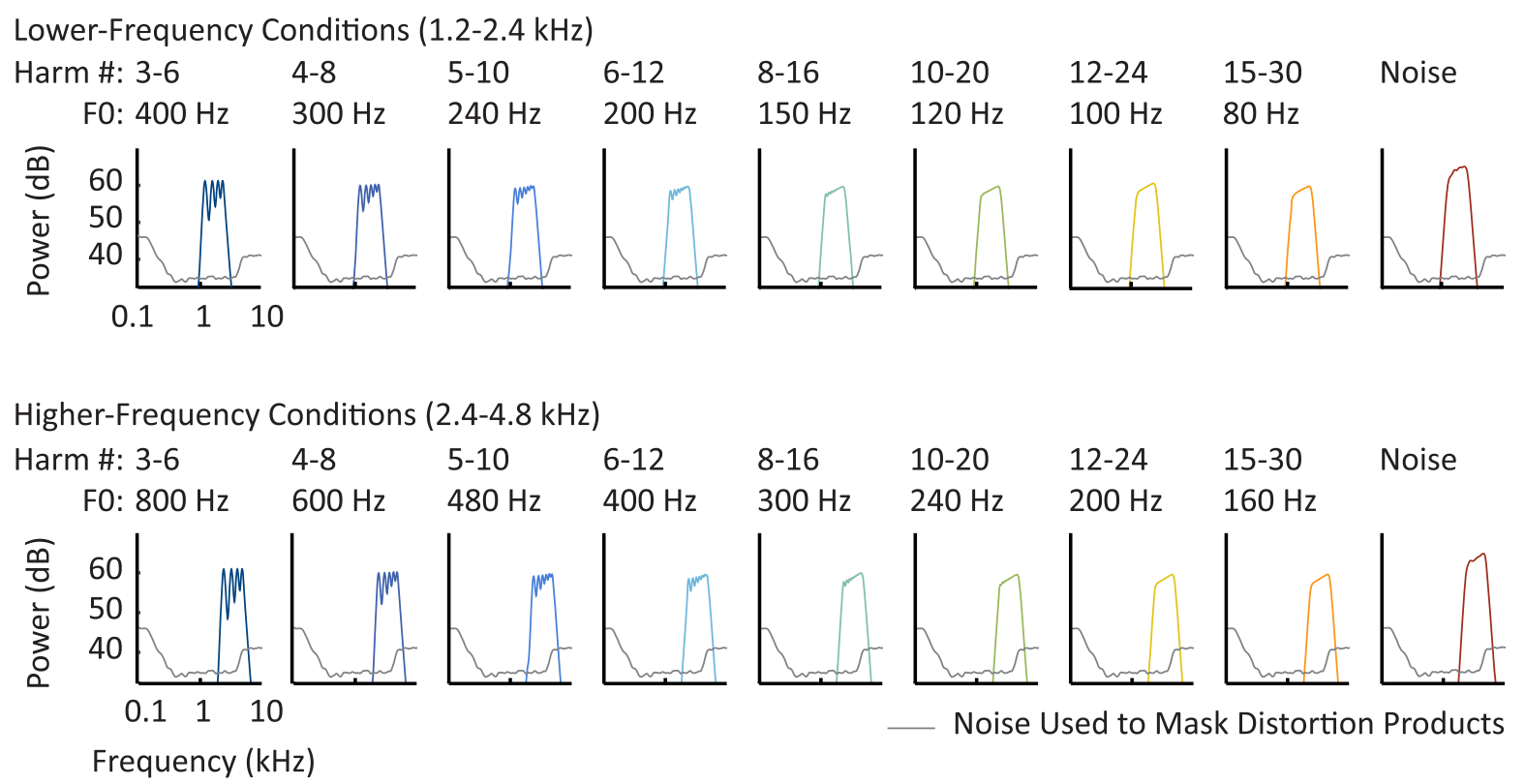

\section{B Schematic of Stimulus Presentation}

Block

5 stimuli, $17 \mathrm{sec}$

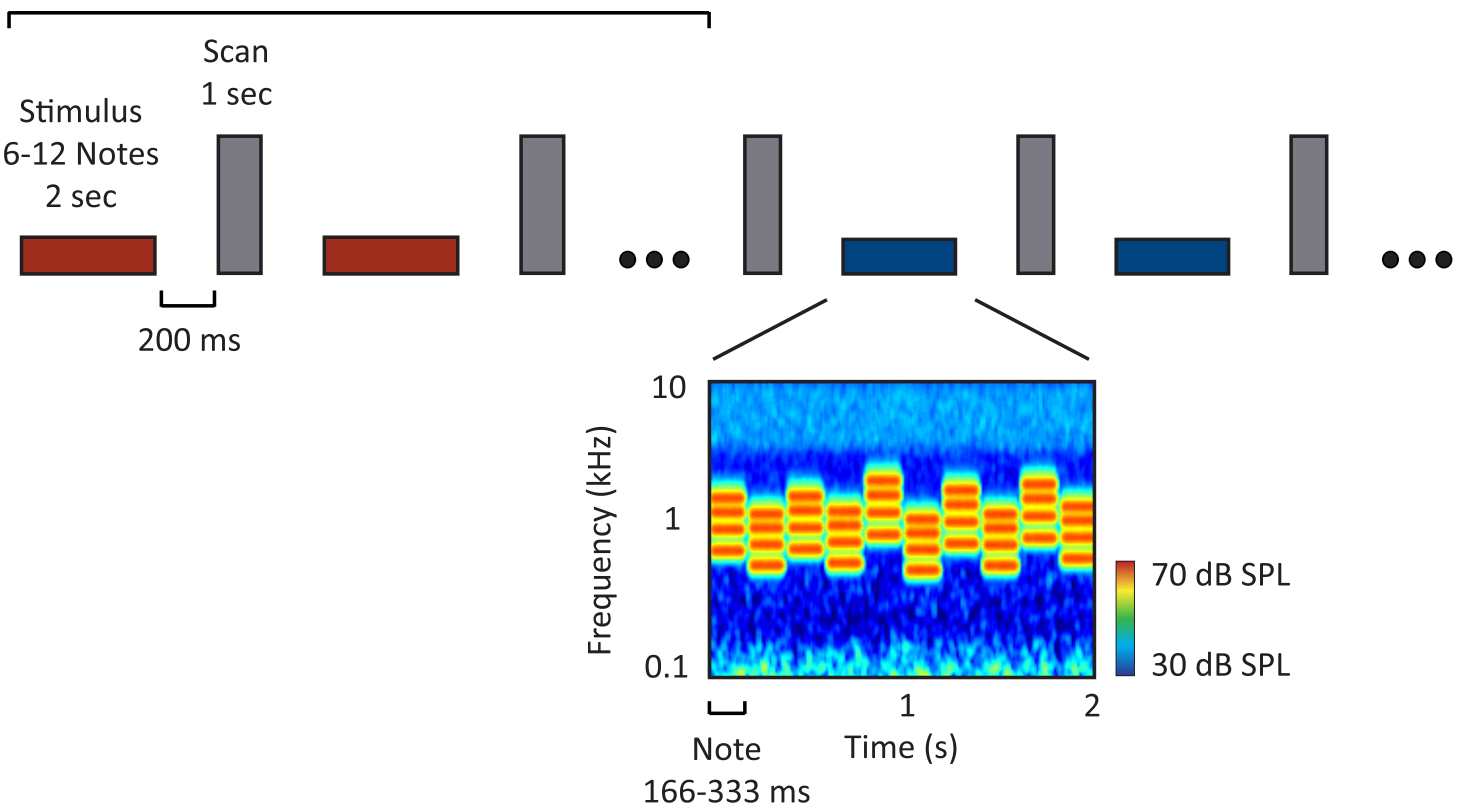

Figure 2. Study design. $\boldsymbol{A}$, Simulated excitation patterns for example notes from each harmonic and noise condition in the parametric resolvability experiment (see Materials and Methods for details). Two frequency ranges (rows) were crossed with eight sets of harmonic numbers (columns) to yield 16 harmonic conditions. As can be seen, the excitation peaks decrease in definition with increasing harmonic number (left to right), but are not greatly affected by changes in F0 or absolute frequency that do not alter harmonic number (top to bottom). Complexes with harmonics below the eighth are often considered resolved because they are believed to produce excitation peaks and troughs separated by at least $1 \mathrm{~dB}$ (Micheyl et al., 2010 ). For each frequency range, a spectrally matched noise control was included as a nonpitch baseline (the spectrum level of the noise was increased by $5 \mathrm{~dB}$ relative to the harmonic conditions to equalize perceived loudness). $\boldsymbol{B}$, Schematic of stimulus presentation. Stimuli (denoted by horizontal bars) were presented in a block design, with five stimuli from the same condition presented successively in each block (red and blue indicate different conditions). Each stimulus was 2 s in duration and included 6-12 different notes. After each stimulus, a single scan was collected (vertical, gray bars). To minimize note-to-note adaptation, notes within a given stimulus/condition varied in their frequency and F0 within a limited range specified for that condition (such that harmonic number composition did not vary across the notes of a condition). A cochleogram for an example stimulus is shown at the bottom (plotting the magnitude of the cochlear response as a function of frequency and time, computed using a gammatone filterbank). Masking noise is visible at low and high frequencies. 
Resolvability is believed to be determined by the spacing of harmonics relative to cochlear filter bandwidths, a key consequence of which is that the resolvability of a harmonic depends primarily on its number in the harmonic sequence (i.e., the ratio between its frequency and the F0) rather than on absolute frequency or absolute F0 (Figs. 1A, 2A; Houtsma and Smurzinski, 1990; Carlyon and Shackleton, 1994; Shackleton and Carlyon, 1994; Plack et al., 2005). This is because auditory filter bandwidths increase with the center frequency of the filter on a linear frequency scale and are relatively constant when plotted on a logarithmic scale (although not perfectly constant, see Glasberg and Moore, 1990), whereas harmonics are separated by a fixed amount on a linear scale and thus grow closer together on a logarithmic scale as harmonic number increases. As a result, low-numbered harmonics, which fall under filters that are narrow relative to the harmonic spacing, produce detectable peaks in the cochlea's excitation pattern, whereas high-numbered harmonics do not (Figs. 1A, 2A). In contrast, both resolved and unresolved harmonics produce periodic fluctuations in the temporal response of individual cochlear nerve fibers (Fig. $1 B$ ), because multiple harmonics passed through the same frequency channel produce beating at the frequency of the F0.

The key piece of evidence that resolved harmonics are important for pitch perception is that complexes with only high-numbered harmonics produce a weaker pitch percept than complexes with low-numbered harmonics (Houtsma and Smurzinski, 1990; Shackleton and Carlyon, 1994). Behaviorally, this effect manifests as a sharp increase in pitch discrimination thresholds when the lowest harmonic in a complex (generally the most resolved component) is higher than approximately the eighth harmonic (Bernstein and Oxenham, 2005). This "cutoff" point is relatively insensitive to the absolute frequency range or F0 of the complex, as can be demonstrated experimentally by fixing the stimulus frequency range and varying the F0 or, conversely, by fixing the F0 and varying the frequency range (Shackleton and Carlyon, 1994; Penagos et al., 2004).

\section{General methods}

Stimuli for parametric resolvability manipulation. Because controlling for stimulus frequency content is particularly important in studying auditory neural responses, we presented harmonics within a fixed absolute frequency range when manipulating resolvability (Fig. $2 A$, each row has the same frequency range). The resolvability of each harmonic complex was varied by changing the F0, which alters the harmonic numbers present within the fixed frequency range (Fig. $2 A$, harmonic numbers increase from left to right). To control for effects of F0, we tested two different frequency ranges, presenting the same harmonic numbers with different F0s (Fig. 2A; top and bottom rows have the same harmonic numbers, but different F0s and frequency ranges). Behaviorally, we expected that pitch discrimination thresholds would be high (indicating poor performance) for complexes in which the lowest component was above the eighth harmonic and that this cutoff point would be similar across the two frequency ranges (Shackleton and Carlyon, 1994; Bernstein and Oxenham, 2005). The goal of this part of the study was to test whether pitch-sensitive brain regions preferentially respond to harmonics that are resolved and, if so, whether they exhibit a nonlinear response cutoff as a function of harmonic number, similar to that measured behaviorally.

There were 18 conditions in this first part of the study: 16 harmonic conditions and two noise controls. Across the 16 harmonic conditions, we tested eight sets of harmonic numbers (Fig. $2 A$, columns) in two different frequency ranges (Fig. $2 A$, rows). The noise conditions were separately matched to the two frequency ranges used for the harmonic conditions.

Stimuli were presented in a block design with five stimuli from the same condition presented successively in each block (Fig. 2B). Each stimulus lasted $2 \mathrm{~s}$ and was composed of several "notes" of equal duration. Notes were presented back to back with $25 \mathrm{~ms}$ linear ramps applied to the beginning and end of each note. The number of notes per stimulus varied (with equal probability, each stimulus had $6,8,10$, or 12 notes) and the duration of each note was equal to the total stimulus duration $(2 \mathrm{~s})$ divided by the number of notes per stimulus. After each stimulus, a single scan/volume was collected ("sparse sampling"; Hall et al., 1999). Each scan acquisition lasted $1 \mathrm{~s}$, with stimuli presented during the $2.4 \mathrm{~s}$ interval between scans (for a total TR of $3.4 \mathrm{~s}$ ). A fixed background noise was present throughout the $2.4 \mathrm{~s}$ interscan interval to mask distortion products (see details below).

To minimize note-to-note adaptation, we varied the frequency range and F0 of notes within a condition over a 10-semitone range. The F0 and frequency range covaried such that the power at each harmonic number remained the same, ensuring that all notes within a condition would be similarly resolved (Fig. 2B, cochleogram). This was implemented by first sampling an F0 from a uniform distribution on a log scale, then generating a complex tone with a full set of harmonics, and then band-pass filtering this complex in the spectral domain to select the desired set of harmonic numbers. The filter cutoffs for the notes within a condition were thus set relative to the note harmonics. For example, the filter cutoffs for the most resolved condition were set to the third and sixth harmonic of each note's F0 (e.g., a note with a $400 \mathrm{~Hz} F 0$ would have a passband of $1200-2400 \mathrm{~Hz}$ and a note with $375 \mathrm{~Hz}$ F0 would have a passband of $1125-2250 \mathrm{~Hz}$ ).

The 8 passbands for each frequency range corresponded to harmonics $3-6,4-8,5-10,6-12,8-16,10-20,12-24$, and 15-30. For the 8 lower frequency conditions, the corresponding mean F0s were $400 \mathrm{~Hz}$ (for harmonics 3-6), $300 \mathrm{~Hz}$ (for harmonics 4-8), $240 \mathrm{~Hz}$ (5-10), $200 \mathrm{~Hz}$ (6-12), $150 \mathrm{~Hz}(8-16), 120 \mathrm{~Hz}(10-20), 100 \mathrm{~Hz}(12-24)$, and $80 \mathrm{~Hz}$ (15-30). These conditions spanned the same frequency range because the product of the mean $\mathrm{F} 0$ and the lowest/highest harmonic number of each passband was the same for all 8 conditions (e.g., $400^{*}[36]=300^{*}[48]=$ $240^{*}[510]$ etc.). For the 8 conditions with a higher-frequency range, the corresponding F0s were an octave higher $(800 \mathrm{~Hz}$ for harmonics 3-6, 600 $\mathrm{Hz}$ for harmonics 4-8, etc.).

For all conditions, harmonics were added in sine phase, and the skirts of the filter sloped downward at $75 \mathrm{~dB}$ /octave (i.e., a linear decrease in amplitude for $\mathrm{dB}$-amplitude and log-frequency scales). We chose to manipulate the harmonic content of each note via filtering (as opposed to including a fixed number of equal-amplitude components) to avoid sharp spectral boundaries, which might otherwise provide a weakly resolved pitch cue, perhaps due to lateral inhibition (Small and Daniloff, 1967; Fastl, 1980).

The two noise conditions were matched in frequency range to the harmonic conditions (with one noise condition for each of the two frequency ranges). For each noise note, a "reference frequency" (the analog of an F0) was sampled from a uniform distribution on a log-scale and Gaussian noise was then band-pass filtered relative to this sampled reference frequency. For the lower frequency noise condition, the mean reference frequency was $400 \mathrm{~Hz}$ and the passband was set to 3 and 6 times the reference frequency. For the high-frequency noise condition, the mean reference frequency was increased by an octave to $800 \mathrm{~Hz}$.

Figure $2 A$ shows simulated excitation patterns for a sample note from each condition (for illustration purposes, F0s and reference frequencies are set to the mean for the condition). These excitation patterns were computed from a standard gammatone filter bank (Slaney, 1998; Ellis, 2009) designed to give an approximate representation of the degree of excitation along the basilar membrane of the cochlea. It is evident that harmonics above the eighth do not produce discernible peaks in the excitation pattern.

Harmonic notes were presented at $67 \mathrm{~dB}$ SPL and noise notes were presented at $72 \mathrm{~dB}$ SPL; the $5 \mathrm{~dB}$ increment was chosen based on pilot data suggesting that this increment was sufficient to equalize loudness between harmonic and noise notes. The sound level of the harmonic conditions was chosen to minimize earphone distortion (see below).

Measuring and controlling for distortion products. An important challenge when studying pitch is to control for cochlear distortion products (DPs) - frequency components introduced by nonlinearities in the cochlea's response to sound (Goldstein, 1967). This is particularly important for examining the effects of resolvability because distortion can introduce low-numbered harmonics not present in the original stimulus. Although an unresolved pitch stimulus might be intended to convey purely temporal pitch information, the DPs generated by an unresolved stimulus can in principle act as a resolved pitch cue. To eliminate the 
potentially confounding effects of distortion, we used colored noise to energetically mask distortion products, a standard approach in both psychophysical and neuroimaging studies of pitch perception (Licklider, 1954; Houtsma and Smurzinski, 1990; Penagos et al., 2004; Hall and Plack, 2009).

Cochlear DPs were estimated psychophysically using the beatcancellation technique (Goldstein, 1967; Pressnitzer and Patterson, 2001). This approach takes advantage of the fact that cochlear DPs are effectively pure tones and can therefore be cancelled using another pure tone at the same amplitude and opposite phase as the DP. The effect of cancellation is typically made more salient with the addition of a second tone designed to produce audible beating with the DP when present (i.e., not cancelled). Because the perception of beating is subtle in this paradigm, the approach requires some expertise, and thus DP amplitudes were estimated by author S.N.-H. in both ears and replicated in a few conditions by two other laboratory members. We used the maximum measured DP across the two ears of S.N.-H. as our estimate of the distortion spectrum. The DP amplitudes we measured for our stimuli were similar to those reported by Pressnitzer and Patterson (2001) for an unresolved harmonic complex.

We found that the amplitude of cochlear DPs primarily depended on the absolute frequency of the DP (as opposed to the harmonic number of the DP relative to the primaries) and that lower-frequency DPs were almost always higher in amplitude (a pattern consistent with the results of Pressnitzer and Patterson, 2001, and likely in part explained by the fact that the cancellation tone, but not the DP, is subject to the effects of middle ear attenuation). As a conservative estimate of the distortion spectrum (e.g., the maximum cochlear DP generated at each frequency), we measured the amplitude of DPs generated at the F0 for a sample harmonic complex from every harmonic condition (with the F0 set to the mean of the condition) and used the maximum amplitude at each absolute frequency to set our masking noise at low frequencies.

Distortion can also arise from nonlinearities in sound system hardware. In particular, the MRI-compatible Sensimetrics earphones we used to present sound stimuli in the scanner have higher levels of distortion than most high-end audio devices due to the piezo-electric material used to manufacture the earphones (according to a personal communication with the manufacturers of Sensimetrics earphones). Sensimetrics earphones have nonetheless become popular in auditory neuroimaging because (1) they are MRI-safe, (2) they provide considerable sound attenuation via screw-on earplugs (necessary because of the loud noise produced by most scan sequences), and (3) they are small enough to use with modern radio-frequency coils that fit snugly around the head (here we use a 32-channel coil). To ensure that earphone distortion did not influence our results, we presented sounds at a moderate sound level (67 dB SPL for harmonic conditions) that produced low earphone distortion, and we exhaustively measured all of the earphone DPs generated by our stimuli (details below). We then adjusted our masking noise to ensure that both cochlear and earphone DPs were energetically masked.

Distortion product masking noise. The masking noise was designed to be at least $10 \mathrm{~dB}$ above the masked threshold of any cochlear or earphone DP, i.e. the level at which the DP would have been just detectable. This relatively conservative criterion should render all DPs inaudible. To accomplish this while maintaining a reasonable signal-to-noise ratio, we used a modified version of threshold-equalizing noise (TEN) (Moore et al., 2000) that was spectrally shaped to have greater power at frequencies with higher-amplitude DPs. The noise took into account the DP level, the auditory filter bandwidth at each frequency, and the efficiency with which a tone can be detected at each frequency. For frequencies between $400 \mathrm{~Hz}$ and $5 \mathrm{kHz}$, both cochlear and earphone DPs were low in amplitude (never exceeding $22 \mathrm{~dB}$ SPL) and the level of the TEN noise was set to mask pure tones below $32 \mathrm{~dB}$ SPL. At frequencies below $400 \mathrm{~Hz}$, cochlear DPs always exceeded earphone DPs and increased in amplitude with decreasing frequency. We therefore increased the spectrum of the TEN noise at frequencies below $400 \mathrm{~Hz}$ by interpolating the cochlear distortion spectrum (measured psychophysically as described above) and adjusting the noise such that it was at least $10 \mathrm{~dB}$ above the masked threshold of any DP. For frequencies above $5 \mathrm{kHz}$, we increased the level of the TEN noise by $6 \mathrm{~dB}$ to mask Sensimetrics earphone DPs, which reached a peak level of $28 \mathrm{~dB}$ SPL at $5.5 \mathrm{kHz}$ (due to a high-frequency resonance in the earphone transfer function). This $6 \mathrm{~dB}$ increment was implemented with a gradual $15 \mathrm{~dB}$ per octave rise (from 3.8 to $5 \mathrm{kHz}$ ). The excitation spectrum of the masking noise can be seen in Figure $2 \mathrm{~A}$ (gray lines).

Earphone calibration and distortion measurements. We calibrated stimulus levels using a Svantek 979 sound meter attached to a G.R.A.S. microphone with an ear and cheek simulator (Type 43-AG). The transfer function of the earphones was measured using white noise and was inverted to present sounds at the desired level. Distortion measurements were made with the same system. For each harmonic condition in the experiment, we measured the distortion spectrum for 11 different F0s spaced 1 semitone apart (to span the 10-semitone F0 range used for each condition). For each note, DPs were detected and measured by computing the power spectrum of the waveform recorded by the sound meter and comparing the measured power at each harmonic to the expected power based on the input signal and the transfer function of the earphones. Harmonics that exceeded the expected level by $5 \mathrm{~dB}$ SPL were considered DPs. Repeating this procedure across all notes and conditions produced a large collection of DPs, each with a specific frequency and power. At the stimulus levels used in our experiments, the levels of earphone distortion were modest. Below $5 \mathrm{kHz}$, the maximum DP measured occurred at $1.2 \mathrm{kHz}$ and had an amplitude of $22 \mathrm{~dB}$ SPL. Above $5 \mathrm{kHz}$, the maximum DP measured was $28 \mathrm{~dB}$ SPL and occurred at $5.5 \mathrm{kHz}$. We found that higher stimulus levels produced substantially higher earphone distortion for stimuli with a peaked waveform, such as the unresolved pitch stimuli used in this study, and such levels were avoided for this reason.

Tonotopy stimuli. We measured tonotopy using pure tones presented in one of six different frequency ranges spanning a six-octave range, similar to the approach used by Humphries et al. (2010). The six frequency ranges were presented in a block design using the same approach as that for the harmonic and noise stimuli. For each note, tones were sampled from a uniform distribution on a log scale with a 10 -semitone range. The mean of the sampling distribution determined the frequency range of each condition and was set to $0.2,0.4,0.8,1.6,3.2$, and $6.4 \mathrm{kHz}$. There were more notes per stimulus for the pure tone stimuli than for the harmonic and noise stimuli (with equal probability, a stimulus contained $16,20,24$, or 30 notes), because in pilot data we found that frequencyselective regions responded most to fast presentation rates. The sound level of the pure tones was set to equate their perceived loudness in terms of sones ( 8 sones, $72 \mathrm{~dB}$ SPL at $1 \mathrm{kHz}$ ), using a standard loudness model (Glasberg and Moore, 2006).

Stimuli for efficient pitch localizer. Based on the results of our parametric resolvability manipulation, we designed a simplified set of pitch and noise stimuli to localize pitch-responsive brain regions efficiently in each subject while minimizing earphone distortion. Specifically, our pitch localizer consisted of a resolved pitch condition contrasted with a frequency-matched noise control. We chose to use resolved harmonics exclusively because they produced a much higher response in all cortical pitch regions than did unresolved harmonics. Moreover, the use of a single resolved condition allowed us to design a harmonic stimulus that produced very low Sensimetrics earphone distortion even at higher sound levels (achieved by minimizing the peak factor of the waveform and by presenting sounds in a frequency range with low distortion). Given the increasing popularity of Sensimetrics earphones, this localizer may provide a useful tool for identifying pitch-sensitive regions in future research (and can be downloaded from our website). To assess the effectiveness of this localizer, we used it to identify pitch-sensitive voxels in each subject and compared the results with those obtained using the resolved harmonic and noise stimuli from the main experiment. The results of this analysis are described at the end of Part 2 in the Results. We also used the localizer to identify pitch-sensitive voxels in the four subjects who participated in a follow-up scan.

Harmonic and noise notes for the localizer stimuli were generated using the approach described previously for our parametric experimental 
stimuli and were also presented in a block design. The mean F0 of the harmonic localizer conditions was $333 \mathrm{~Hz}$ and the passband included harmonics 3 through 6 (equivalent to the most resolved condition from the parametric manipulation in Part 1 of this study). Harmonic notes were presented at $75 \mathrm{~dB}$ SPL and noise notes were presented at $80 \mathrm{~dB}$ SPL (instead of the lower levels of 67 and $72 \mathrm{~dB}$ SPL used in the parametrically varied stimuli; we found in pilot experiments that higher presentation levels tend to produce higher BOLD responses). Harmonics were added in negative Schroeder phase (Schroeder, 1970) to further reduce earphone DPs, which are largest for stimuli with a high peak factor (perceptually, phase manipulations have very little effect for a resolved pitch stimulus because the individual harmonics are filtered into distinct regions of the cochlea). Cochlear DPs at the F0 and second harmonic were measured for complexes with three different F0s $(249$, 333, and $445 \mathrm{~Hz}$, which corresponded to the lowest, highest, and mean F0 of the harmonic notes), and modified TEN noise was used to render DPs inaudible. The spectrum of the TEN noise was set to ensure that DPs were at least $15 \mathrm{~dB}$ below their masked threshold (an even more conservative criterion than that used in Part 1 of the study). Specifically, below $890 \mathrm{~Hz}$ (the maximum possible frequency of the second harmonic), the level of the TEN noise was set to mask DPs up to $40 \mathrm{~dB}$ SPL. Above $890 \mathrm{~Hz}$, the noise level was set to mask DPs up to $30 \mathrm{~dB}$ SPL. The $10 \mathrm{~dB}$ decrement above $890 \mathrm{~Hz}$ was implemented with a gradual $15 \mathrm{~dB}$ per octave fall-off from $890 \mathrm{~Hz}$ to $1.4 \mathrm{kHz}$. Earphone DPs never exceeded $15 \mathrm{~dB}$ SPL for these stimuli.

Participants. Twelve individuals participated in the fMRI study (4 male, 8 female, all right-handed, ages 21-28 years, mean age 24 years). Eight individuals from the fMRI study also participated in a behavioral experiment designed to measure pitch discrimination thresholds for the same stimuli. To obtain robust tonotopy and pitch maps in a subset of individual subjects, we rescanned four of the 12 subjects in a follow-up session.

Subjects were non-musicians (with no formal training in the 5 years preceding the scan), native English speakers, and had self-reported normal hearing. Pure-Tone detection thresholds were measured in all participants for frequencies between $125 \mathrm{~Hz}$ and $8 \mathrm{kHz}$. Across all frequencies tested, 7 subjects had a maximum threshold at or below $20 \mathrm{~dB}$ HL, 3 subjects had a maximum threshold of $25 \mathrm{~dB}$ HL, and 1 subject had a maximum threshold of $30 \mathrm{~dB}$ HL. One subject had notable highfrequency hearing loss in the right ear (with thresholds of $70 \mathrm{~dB} H \mathrm{HL}$ and higher for frequencies $6 \mathrm{kHz}$ and above; left ear thresholds were $<20 \mathrm{~dB}$ $\mathrm{HL}$ at all frequencies and right ear thresholds were at or below $30 \mathrm{~dB}$ for all other frequencies). Neural data from this subject was included in our fMRI analyses, but the inclusion or exclusion of their data did not qualitatively alter the results. This subject did not participate in the behavioral experiment. Two additional subjects (not included in the 12 described above) were excluded from the study either because not enough data were collected (due to a problem with the scanner) or because the subject reported being fatigued throughout the experiment. In the latter case, the decision to exclude the subject was made before analyzing their data to avoid any potential bias. The study was approved by the Committee On the Use of Humans as Experimental Subjects (COUHES) at the Massachusetts Institute of Technology (MIT), and all participants gave informed consent.

Procedure. Scanning sessions lasted $2 \mathrm{~h}$ and were composed of multiple functional "runs," each lasting 6-7 min. Two types of runs were used in the study. The first type ("resolvability runs") included all of the harmonic and noise stimuli for our parametric resolvability manipulation (i.e., all of the conditions illustrated in Fig. 2A). The second type ("tonotopy runs") included all of the tonotopy stimuli as well as the pitch and noise conditions for the efficient pitch localizer. Runs with excessive head motion or in which subjects were overly fatigued were discarded before analysis. Not including discarded runs, each subject completed 7-8 resolvability runs and 3 tonotopy runs. The 4 subjects who participated in a second scanning session completed 10-12 tonotopy runs (these extra 7-9 runs were not included in group analyses across all 12 subjects to avoid biasing the results). Excessive motion was defined as at least 20 time points whose average deviation (Jenkinson, 1999) from the previous time point exceeded $0.5 \mathrm{~mm}$. Fatigue was obvious due a sudden drop in re- sponse rates on the repetition-detection task (e.g., a drop from a $90 \%$ response rate to a response rate $<50 \%$, see below). Across all 12 subjects, a total of two runs were discarded due to head motion and two runs were discarded due to fatigue. In addition, data from a second scanning session with a fifth subject was excluded (before being analyzed) because response rates were very low in approximately half of the runs.

Each resolvability run included one stimulus block for each of the 18 conditions shown in Figure $2 A$ (each block lasting $17 \mathrm{~s}$ ) as well as four "null" blocks with no sound stimulation (also $17 \mathrm{~s}$ ) to provide a baseline (each run included 114 scans and was $385 \mathrm{~s}$ in total duration). Each tonotopy run included two stimulus blocks for each of the six pure-tone conditions, four stimulus blocks for each of the harmonic and noise conditions from the pitch localizer, and five null blocks (129 scans, $436 \mathrm{~s}$ ). The order of stimulus and null blocks was counterbalanced across runs and subjects such that, on average, each condition was approximately equally likely to occur at each point in the run and each condition was preceded equally often by every other condition in the experiment. After each run, subjects were given a short break $(\sim 30 \mathrm{~s})$.

To help subjects attend equally to all of the stimuli, subjects performed a "1-back" repetition detection task (responding whenever successive $2 \mathrm{~s}$ stimuli were identical) across stimuli in each block for both resolvability and tonotopy runs. Each block included four unique stimuli and one back-to-back repetition (five stimuli per block). Each of the unique stimuli had a different number of notes. Stimuli were never repeated across blocks. Performance on the 1-back task was high for all of the harmonic and noise conditions in the experiment (hit rates between $84 \%$ and $93 \%$ and false alarm rates between $0 \%$ and $2 \%)$. Hit rates for the pure-tone conditions varied from $71 \%(6.4 \mathrm{kHz}$ condition $)$ to $89 \%$ (1.6 kHz condition).

Data acquisition. All data were collected using a 3T Siemens Trio scanner with a 32-channel head coil (at the Athinoula A. Martinos Imaging Center of the McGovern Institute for Brain Research at MIT). T1weighted structural images were collected for each subject ( $1 \mathrm{~mm}$ isotropic voxels). Each functional volume (e.g., a single whole-brain acquisition) comprised 15 slices oriented parallel to the superior temporal plane and covering the portion of the temporal lobe superior to and including the superior temporal sulcus ( $3.4 \mathrm{~s}$ TR, $1 \mathrm{~s} \mathrm{TA}, 30 \mathrm{~ms}$ TE, 90 degree flip angle; the first 5 volumes of each run were discarded to allow for T1 equilibration). Each slice was $4 \mathrm{~mm}$ thick and had an in-plane resolution of $2.1 \mathrm{~mm} \times 2.1 \mathrm{~mm}(96 \times 96$ matrix, $0.4 \mathrm{~mm}$ slice gap $)$.

Preprocessing and regression analyses. Preprocessing and regression analyses were performed using FSL 4.1.3 and the FMRIB software libraries (Analysis Group, FMRIB, Oxford; Smith et al., 2004). Functional images were motion corrected, spatially smoothed with a $3 \mathrm{~mm}$ FWHM kernel, and high-pass filtered (250 s cutoff). Each run was fit with a general linear model (GLM) in the native functional space. The GLM included a separate regressor for each stimulus condition (modeled with a gamma hemodynamic response function) and six motion regressors (three rotations and three translations). Statistical maps from this within-run analysis were then registered to the anatomical volume using FLIRT (Jenkinson and Smith, 2001) followed by BBRegister (Greve and Fischl, 2009).

Surface-based analyses. For anatomical and tonotopy analyses, these within-run maps were resampled to the cortical surface, as estimated by Freesurfer (Fischl et al., 1999), and combined across runs within subjects using a fixed effects analysis. For simplicity, we use the term "voxel" in describing all of our analyses rather than switching to the slightly more accurate term "vertex" when discussing surface-based analyses (vertices refer to a $2 \mathrm{D}$ point on the cortical surface).

\section{Analyses for Part 1: Cortical responses to resolved and unresolved harmonics}

ROI analyses of cortical pitch responses. To test the response of pitchsensitive brain regions, we first identified voxels in the superior temporal plane of each subject that responded more to all 16 harmonic conditions compared with the two frequency-matched noise conditions (the localize phase) and then measured the response of these voxels in independent (left-out) data to all 18 conditions (the test phase; Fig. $3 A, B, D$ ). Importantly, this localizing procedure was unbiased with respect to resolvabil- 
ity effects because it included both resolved and unresolved harmonics. We considered only voxels that fell within an anatomical constraint region encompassing the superior temporal plane. This constraint region spanned HG, the planum temporale, and the planum polare and was defined as the combination of the 5 ROIs used in the anatomical analyses (Fig. 4A). We chose the superior temporal plane as a constraint region because almost all prior reports of pitch responses have focused on this region and because, in practice, we rarely observed consistent pitch responses outside of the superior temporal plane.

We implemented this localize-and-test procedure using a simple leaveone-out design performed across runs, similar to the approach typically used in classification paradigms such as multivoxel pattern analysis (Norman et al., 2006). With 3 runs, for example, there would be 3 localize/test pairs: localize with runs 2 and 3 and test with run 1, localize with runs 1 and 3 and test with run 2, and localize with runs 1 and 2 and test with run 3 . For each localize-test pair, we identified pitch-sensitive voxels using data from every run except the test run and we then measured the mean response of these localized voxels to all 18 harmonic and noise conditions in the test run. Each run therefore provided an unbiased sample of the response to every condition and we averaged responses across runs to derive an estimate of the response to each condition in each subject.

For the localize phase, we computed subject-specific significance maps using a fixed-effects analysis across all runs except the test run and selected the $10 \%$ of voxels with the most significant response within the anatomical constraint region (due to variation in brain size, the total volume of these voxels ranged from 1.1 to $1.6 \mathrm{~cm}^{3}$ across subjects). We chose to use a fixed percentage of voxels instead of a fixed significance threshold (e.g., $p<0.001$ ) because differences in significance values across subjects are often driven by generic differences in signal-to-noise that are unrelated to neural activity (such as amount of motion). In the test phase, we then measured the response of these voxels in independent data.

For the test phase, we measured the mean response time course for each condition by averaging the signal from each block from that condition. Time courses were then converted to percent signal change by subtracting and dividing by the mean time course for the null blocks (pointwise, for each time point of the block). The mean response to each condition was computed by averaging the response of the second through fifth time points after the onset of each block. Response time courses are shown in Figure $3 A$ and the gray area indicates the time points included in the average response for each condition.

Behavioral pitch discrimination experiment. To assess the perceptual effects of our resolvability manipulation, we measured pitch discrimination thresholds for all of the harmonic conditions in the study for a subset of eight subjects from the imaging experiment (Fig. 3C,E). Subjects were asked to judge which of two sequentially presented notes was higher in pitch. We used an adaptive procedure (3-up, 1-down) to estimate the F0 difference needed to achieve $79 \%$ accuracy (Levitt, 1971). Subjects completed between one and three adaptive sequences for each of the 16 harmonic conditions tested in the imaging experiment (five subjects completed two sequences, two subjects completed three sequences, and one subject completed a single sequence). In each sequence, we measured 12 reversals and the threshold for a given subject and condition was defined as the geometric mean of the last eight reversal points of each sequence. The frequency difference at the start of the sequence was set to $40 \%$ and this difference was updated by a factor of 1.414 (square root of 2 ) for the first 4 reversals and by a factor of 1.189 (fourth root of 2 ) for the last 8 reversals.

The "notes" used as stimuli in the behavioral experiment were designed to be as similar as possible to the notes used in the imaging experiments, subject to the constraints of a discrimination experiment. To encourage subjects to rely on pitch cues as opposed to overall frequency cues, each pair of notes on a given trial was filtered with the same filter, such that the frequency range was constant across the notes of a single trial. This was partially distinct from the imaging experiment, in which each note had a unique filter defined relative to its F0, but was necessary to isolate F0 discrimination behaviorally. The filter cutoffs for a note pair were set relative to the center frequency (geometric mean) of the F0s for the two notes using the same procedure as that for the imaging experiment. Center frequencies were varied across trials (uniformly sampled from $\pm 10 \%$ of the mean F0 for that condition) to encourage subjects to compare notes within a trial instead of making judgments based on the distribution of frequencies across trials (e.g., by judging whether a note is higher or lower in frequency than the notes in previous trials). The mean F0 of each condition was the same as that used in the imaging experiment. Each note was $333 \mathrm{~ms}$ in duration and pairs of notes were separated by a $333 \mathrm{~ms}$ interstimulus interval. We used the same MRI-compatible earphones to measure behavioral thresholds and the same colored noise to mask cochlear and earphone distortion. Masking noise was gated on $200 \mathrm{~ms}$ before the onset of the first note and was gated off $200 \mathrm{~ms}$ after the offset of the second note ( $25 \mathrm{~ms}$ linear ramps). Conditions with the same frequency range were grouped into sections and participants were encouraged to take a break after each section. The order of low- and highfrequency sections was counterbalanced across subjects and the order of conditions within a section was randomized.

Analyses for Part 2: Anatomical location of cortical pitch responses Anatomical ROI analysis. To examine the anatomical locations of pitchsensitive brain regions, we divided the superior temporal plane into five nonoverlapping anatomical ROIs (based on prior literature, as detailed below; Fig. $4 A$ ) and measured the proportion of pitch-sensitive voxels that fell within each region.

We again identified pitch-sensitive voxels as those responding preferentially to harmonic tones compared with noise (the top $10 \%$ of voxels in the superior temporal plane with the most significant response to this contrast) and then measured the number of pitch-sensitive voxels that fell within each subregion of the superior temporal plane (Fig. 4B). Because the 5 anatomical ROIs subdivided our superior temporal plane ROI, this analysis resulted in a 5-bin histogram, which we normalized to sum to 1 for each subject. As a baseline, we also measured the proportion of sound-responsive voxels that fell within each region (Fig. 4C). Soundresponsive voxels were defined by contrasting all 18 stimulus conditions with silence (voxel threshold of $p<0.001$ ). To compare the anatomical distribution of pitch and sound responses directly, we also computed the proportion of sound-responsive voxels in each region that exhibited a pitch response (Fig. 4C, inset).

The five ROIs corresponded to posteromedial HG (TE1.1), middle HG (TE1.0), anterolateral HG (TE1.2), planum temporale (posterior to HG), and planum polare (anterior to HG). The HG ROIs are based on human postmortem histology (Morosan et al., 2001) and the temporal plane ROIs (planum polare and planum temporale) are based on human macroscopic anatomy (distributed in FSL as the Harvard Cortical Atlas; Desikan et al., 2006). To achieve better surface-based alignment and to facilitate visualization, the ROIs were resampled to the cortical surface of the MNI305 template brain (Fig. 4A).

The distribution of pitch responses across these five ROIs suggested that pitch responses might be more concentrated in anterior regions of auditory cortex. To test this idea directly, we performed the same analysis using a set of five novel ROIs that were designed to run along the posterior-to-anterior axis of the superior temporal plane, demarcated so as to each include an equal number of sound-responsive voxels (Figs. $4 D-F$ ). We created these five ROIs by: (1) flattening the cortical surface (Fischl et al., 1999) to create a 2D representation of the superior temporal plane, (2) drawing a line on the flattened surface that ran along the posterior-to-anterior axis of HG, (3) sorting voxels based on their projected location on this line, and (4) grouping voxels into five regions such that, on average, each region would have an equal number of sound-responsive voxels. This final step was implemented by computing a probability map expressing the likelihood that a given voxel would be classified as sound-responsive and then constraining the sum of this probability map to be the same for each region. The main effect of this constraint was to enlarge the size of the most anterior ROI to account for the fact that auditory responses occur less frequently in the most anterior portions of the superior temporal plane. The anatomical ROIs used in the study can be downloaded from our website. 
Anatomical distribution of resolvability effects across auditory cortex. To test whether effects of resolvability are present throughout auditory cortex, we performed the localize-and-test ROI analysis within the five posterior-to-anterior ROIs from the previous analysis (Fig. 5). For a given ROI, we identified the $10 \%$ of voxels (within that ROI) in each subject with the most significant response preference for harmonic tones compared with noise and measured their response to each condition in independent data.

\section{Analyses for Part 3: The tonotopic location of pitch-sensitive brain regions}

The results from Parts 1 and 2 of our study suggested that pitch-sensitive regions are localized to the anterior half of auditory cortex and that pitch responses throughout auditory cortex are driven predominantly by the presence of resolved harmonics. To further clarify the anatomical organization of pitch responses, we computed whole-brain maps of pitch responses and tonotopy. Because these analyses were qualitative, we quantified and validated the main results from these analyses by performing within-subject ROI analyses.

Whole-brain map of pitch responses. For comparison with tonotopy maps, we computed a whole-brain summary figure indicating the proportion of subjects with a pitch response at each point on the cortical surface (Fig. 6A). We focused on pitch responses to conditions with resolved harmonics (defined as having harmonics below the eighth component) because we found in Part 1 that resolved pitch stimuli produced larger pitch responses throughout auditory cortex and thus provide the most robust measure of pitch sensitivity. Pitchsensitive voxels were defined in each subject as the $10 \%$ of voxels in the superior temporal plane that responded most significantly to tones with resolved harmonics compared with noise. For each voxel, we then counted the proportion of subjects in which that voxel was identified as pitch-sensitive. To help account for the lack of exact overlap across subjects in the location of functional responses, analyses were performed on lightly smoothed significance maps $(3 \mathrm{~mm}$ FWHM kernel on the surface; without this smoothing, group maps appear artificially pixelated).

Whole-brain map of best frequency. For each subject, we computed best-frequency maps by assigning each voxel in the superior temporal plane to the pure-tone condition that produced the maximum response, as estimated by the $\beta$-weights from the regression analysis (Fig. 6B). Because not all voxels in a given subject were modulated by frequency, we masked this best-frequency map using the results of a one-way, fixedeffects ANOVA across the six pure-tone conditions $(p<0.05)$. Group tonotopy maps were computing by smoothing ( $3 \mathrm{~mm}$ FWHM) and averaging the individual-subject maps, masking out those voxels that did not exhibit a significant frequency response in at least three subjects (as determined by the one-way ANOVA).

ROI analysis of low-and high-frequency voxels. To further quantify the relationship between pitch responses and frequency tuning, we measured the response of low- and high-frequency-selective voxels to the harmonic and noise conditions (Fig. 6C). Voxels selective for low frequencies were identified in each subject individually as the $10 \%$ of voxels with the most significant response preference for low versus high frequencies (using responses to the tonotopy stimuli: $0.2+0.4+0.8 \mathrm{kHz}>1.6+3.2+6.4$ $\mathrm{kHz})$. High-frequency voxels were defined in the same way using the reverse contrast $(1.6+3.2+6.4 \mathrm{kHz}>0.2+0.4+0.8 \mathrm{kHz})$. We then measured the average response of these voxels to the resolved harmonic and noise conditions used to identify pitch responses in the whole-brain analysis.

Whole-brain map of frequency selectivity. To estimate the distribution of (locally consistent) frequency selectivity across the cortex, we also computed maps plotting the degree of variation in the response of each voxel to the six pure-tone conditions (Fig. 6D). This was quantified as the standard deviation in the response of each voxel to the six pure-tone conditions (estimated from regression $\beta$-weights), divided by the mean response across all six conditions. Because such normalized measures become unstable for voxels with a small mean response (due to the small denominator), we excluded voxels that did not exhibit a significant mean response to the six pure-tone conditions relative to silence $(p<0.001)$ and truncated negative responses to zero. Frequency-selectivity maps were computed for each subject, smoothed ( $3 \mathrm{~mm}$ FWHM), and averaged across subjects. Voxels that did not exhibit a significant sound response in at least three subjects were again excluded.

Statistical comparison of pitch responses and frequency selectivity. To further test the relationship between pitch responses and frequency selectivity, we measured the frequency selectivity of pitch-sensitive voxels as a function of their posterior-to-anterior position (Fig. 6E). As in the whole-brain analysis, pitch-sensitive voxels were identified as the $10 \%$ of voxels in each subject with the most significant response preference for resolved harmonics compared with noise. Frequency selectivity was computed for each pitch-sensitive voxel in each subject individually and was defined as the standard deviation of the response across the six pure-tone conditions divided by the mean. We then grouped voxels into 10 bins (each containing $10 \%$ of all pitchsensitive voxels) based on their posterior-to-anterior position in that subject and averaged selectivity measures within each bin.

Maps of pitch and tonotopy within individual subjects. To demonstrate that the effects we observed at the group level are present in individual subjects, we also present individual subject maps of pitch responses, tonotopy, and frequency selectivity for four subjects who completed an extra scanning session to increase the reliability of their tonotopic maps (Fig. 7). Pitch maps were computed by contrasting responses to resolved harmonics with noise, using data from the "efficient pitch localizer" described earlier (whole-brain maps plot the significance of this contrast). Maps of best-frequency and frequency selectivity were computed using the same approach as the group analyses, except that there was no additional smoothing on the surface and we used a stricter criterion for defining voxels as frequency-selective when computing best-frequency maps $(p<0.001)$. We were able to use a stricter threshold because we had three to four times as much tonotopy data within each subject.

\section{Control experiment: Are resolvability effects dependent on frequency variation?}

One notable feature of our main experiment was that the stimuli varied in pitch and frequency (Figs. $2 B, 8 A$ ). We made this design choice because in pilot experiments we repeatedly found that this variation enhanced the cortical response and thus improves statistical power. However, it is conceivable that frequency/pitch variation could bias cortical responses in favor of resolved harmonics, because notes with sparser spectral representations (e.g., on the cochlea) will tend to overlap less with each other. Less note-to-note spectral overlap for resolved stimuli could reduce the effects of frequency adaptation and inflate responses to resolved harmonics relative to stimuli without resolved spectral peaks for reasons potentially unrelated to their role in pitch perception.

To rule out the possibility that resolvability effects are merely a byproduct of note-to-note frequency/pitch variation, we tested whether neural resolvability effects persist for stimuli with a single, repeated note (Fig. $8 B$, top row). We also measured responses to stimuli with two alternating notes and manipulated the spectral overlap between notes directly (Fig. $8 B$, bottom two rows). This allowed us to test whether differences in note-to-note spectral overlap have any significant effect on the response to resolved and unresolved harmonics. In addition to controlling for frequency adaptation, these experiments help to link our findings more directly with those of prior studies of pitch responses, which have primarily used stimuli with repeated notes (Patterson et al., 2002; Hall and Plack, 2009).

The experiment had a $3 \times 3$ factorial design, illustrated in Figure $8 B$. Each stimulus was composed of either (1) a single, repeated note, (2) two alternating notes designed to minimize spectral overlap in the resolved condition while maximizing overlap in the unresolved condition (twonotes overlapping), or (3) two alternating notes with nonoverlapping frequency ranges (two-notes nonoverlapping). This manipulation was fully crossed with a resolvability/pitch manipulation: each note was either a resolved harmonic complex (harmonics 3-6), an unresolved harmonic complex (harmonics 15-30), or frequency-matched noise.

All nine conditions were designed to span a similar frequency range as the stimuli in the main experiment. For the two-note stimuli with nonoverlapping frequency ranges, the lower-frequency note spanned 1.2-2.4 
$\mathrm{kHz}$ and the higher-frequency note spanned $2.88-5.76 \mathrm{kHz}$, resulting in a $20 \%$ spectral gap between the notes (e.g., $[2.88-2.4] / 2.4=0.2$ ). For resolved complexes, the F0s of the low- and high-frequency notes were 400 and $960 \mathrm{~Hz}$, respectively; for unresolved complexes, the F0s were 80 and $192 \mathrm{~Hz}$, respectively. These same notes were used for the single-note condition, but each note was presented in a separate stimulus.

For the two-note conditions with overlapping frequency ranges, there was a low-frequency and a high-frequency stimulus, each with two alternating notes (Fig. 8B). The notes of each stimulus were separated by 2.49 semitones, an amount that minimized spectral overlap between resolved frequency components ( 2.49 semitones is half of the log distance between the first two resolved frequency components of the notes). The frequency range and F0s of the lower-frequency stimuli straddled the lowerfrequency notes of the nonoverlapping and single-note conditions on a log-scale (F0s and frequency ranges were 1.25 semitones above and below those used for the nonoverlapping stimuli). Similarly, the frequency and F0s of the higher-frequency stimuli straddled the higher-frequency notes of the nonoverlapping and single-note stimuli. For 4 of the 8 subjects scanned, the higher-frequency stimuli had a frequency range and F0 that was $7 \%$ lower than intended due to a technical error. This discrepancy had no obvious effect on the data, and we averaged responses across all eight subjects.

To enable the identification of pitch-sensitive voxels using the localizer contrast from our main experiment, we also presented the resolved (harmonics 3-6), unresolved (harmonics 15-30), and noise stimuli from that experiment. We also included tonotopy stimuli, which allowed us to test whether frequency-selective regions (which might be expected to show the largest effects of frequency adaptation) are sensitive to differences in spectral overlap between resolved and unresolved harmonics.

Participants. Eight individuals participated in the control experiment ( 3 male, 5 female, 7 right-handed, 1 left-handed, age 19-28 years, mean age 24 years). All subjects had self-reported normal hearing and were non-musicians ( 7 subjects had no formal training in the 5 years preceding the scan; 1 subject had taken music classes 3 years before the scan). Subjects had normal hearing thresholds $(<25 \mathrm{~dB} H \mathrm{HL})$ at all tested frequencies ( $125 \mathrm{~Hz}$ to $8 \mathrm{kHz}$ ). One of the eight subjects was also a participant in the main experiment. Three additional subjects (not included in the eight described above) were excluded because of low temporal signalto-noise (tSNR) caused by excessive head motion (two before analysis, and one after). tSNR is a standard measure of signal quality in fMRI analyses (Triantafyllou et al., 2005) and was calculated for each voxel as the mean of the BOLD signal across time divided by the standard deviation. All of the excluded subjects had substantially lower tSNR in the superior temporal plane than nonexcluded subjects (mean tSNR of excluded subjects: $54.5,58.2,59.0$; mean tSNR for nonexcluded subjects: $70.4,71.7,78.2,80.3,81.2,81.8,83.7,92.7)$. A higher percentage of subjects were excluded compared with the main experiment because we used a quieter scanning sequence (described below) that we found to be more sensitive to motion (possibly because of longer-than-normal echo times).

Procedure. The procedure was similar to the main experiment. There were two types of runs: "experimental" runs and "localizer" runs. The nine conditions shown in Figure $8 B$ were scanned in the experimental runs. The harmonic and noise stimuli from the main experiment (used to identify pitch-sensitive regions) were scanned in the localizer runs, as were the tonotopy stimuli.

For the experimental runs, we used a quieter scanning sequence with very little acoustic power at the frequencies of the experimental stimuli (Schmitter et al., 2008; Peelle et al., 2010) to avoid any scanner-induced frequency adaptation. This quieter sequence had a single acoustic resonance at $516 \mathrm{~Hz}$ and an overall level of 75-80 dB SPL (measured using an MRI-compatible microphone), which was further attenuated by screw-on earplugs attached to the Sensimetrics earphones. For the localizer runs, we used the same echoplanar sequence as the main experiment to ensure that our localizer contrasts identified the same regions. We did not use the quiet sequence in the main experiment because it produces a lower-quality MR signal (lower spatial resolution and slower acquisition times for the same spatial coverage), making it difficult to test a large number of conditions.
Each subject completed 10-12 experimental runs and 4-6 localizer runs. Each experimental run consisted of nine stimulus blocks, one for each condition, and three blocks of silence. Each block was $19.5 \mathrm{~s}$ in duration and included 5 scan acquisitions spaced 3.9 s apart. During stimulus blocks, a $2 \mathrm{~s}$ stimulus was presented during a $2.4 \mathrm{~s}$ gap between scan acquisitions. Within a block, stimuli were always composed of the same notes, but the number and duration of the notes varied in the same way as in the main experiment (each stimulus had $6,8,10$, or 12 notes; example stimuli shown in Fig. $8 B$ have 10 notes per stimulus). The order of stimulus and null blocks was counterbalanced across runs and subjects in the same way as the main experiment. Each localizer run consisted of 12 stimulus blocks (one for each of the six tonotopy conditions and two for each resolved, unresolved, and noise condition) and three null blocks. The design of the localizer blocks was the same as the main experiment. For both localizer and experimental runs, subjects performed a 1-back task. Scans lasted $\sim 2 \mathrm{~h}$ as in the main experiment.

Data acquisition. With the exception of the quiet scanning sequence, all of the acquisition parameters were the same as the main experiment. For the quiet scanning sequence, each functional volume comprised 18 slices designed to cover the portion of the temporal lobe superior to and including the superior temporal sulcus $(3.9 \mathrm{~s}$ TR, $1.5 \mathrm{~s}$ TA, 45 ms TE, 90 degree flip angle; the first 4 volumes of each run were discarded to allow for T1 equilibration). Voxels were $3 \mathrm{~mm}$ isotropic $(64 \times 64$ matrix, $0.6 \mathrm{~mm}$ slice gap $)$.

Analysis. We defined pitch-sensitive voxels as the $10 \%$ of voxels in the anterior superior temporal plane with the most significant response preference for resolved harmonics compared with noise (using stimuli from the localizer runs). In contrast to the main experiment, pitch responses were constrained to the anterior half of the superior temporal plane (defined as the three most anterior ROIs from the posterior-to-anterior analysis) because we found in the main experiment that the large of majority of pitch responses (89\%) were located within this region. We also separately measured responses from pitch-sensitive voxels in the middle ROI from the posterior-to-anterior analysis (the most posterior ROI with a substantial number of pitch responses) and the most anterior ROI (pitch-sensitive voxels were again defined as $10 \%$ of voxels with the most significant response preference for resolved harmonics compared with noise in each region). This latter analysis was designed to test whether more anterior pitch regions show a larger response preference for stimuli with frequency variation, as suggested by prior studies (Patterson et al., 2002; Warren and Griffiths, 2003; Puschmann et al., 2010). To test this possibility, we measured the relative difference in response in each ROI to the two-note and one-note conditions using a standard selectivity measure ([two-note - one-note]/[two-note + one-note]) and then compared this selectivity measure across the two ROIs.

For the tonotopy analyses, we focused on regions preferring high frequencies because they are distinct from pitch-sensitive regions (as demonstrated in the main experiment) and because our stimuli spanned a relatively high-frequency range (above $1 \mathrm{kHz}$ ). High-frequency voxels were defined as the $10 \%$ of voxels in the superior temporal plane with the most significant response preference for high $(1.6,3.2,6.4 \mathrm{kHz})$ versus low $(0.2,0.40 .8 \mathrm{kHz})$ frequencies. One of the eight subjects was excluded from the tonotopy analysis because of a lack of replicable high-frequency preferring voxels in left-out test data.

\section{Results \\ Part 1: Cortical responses to resolved and unresolved harmonics}

To assess the response of pitch-sensitive brain regions to spectral and temporal cues, we first identified voxels in each subject that respond more to harmonic complex tones than noise regardless of resolvability (by selecting the 10\% of voxels from anywhere in the superior temporal plane with the most significant response to this contrast). We then measured the response of these voxels to each harmonic and noise condition in independent data. Figure $3 \mathrm{~A}$ plots the average time course across these voxels for each harmonic and noise condition, collapsing across the two frequency ranges tested, and Figure $3 B$ shows the response averaged 


\section{A Response Timecourse of Pitch-Sensitive Voxels}

\section{B Average Response (at Plateau) of Pitch-Sensitive Voxels}

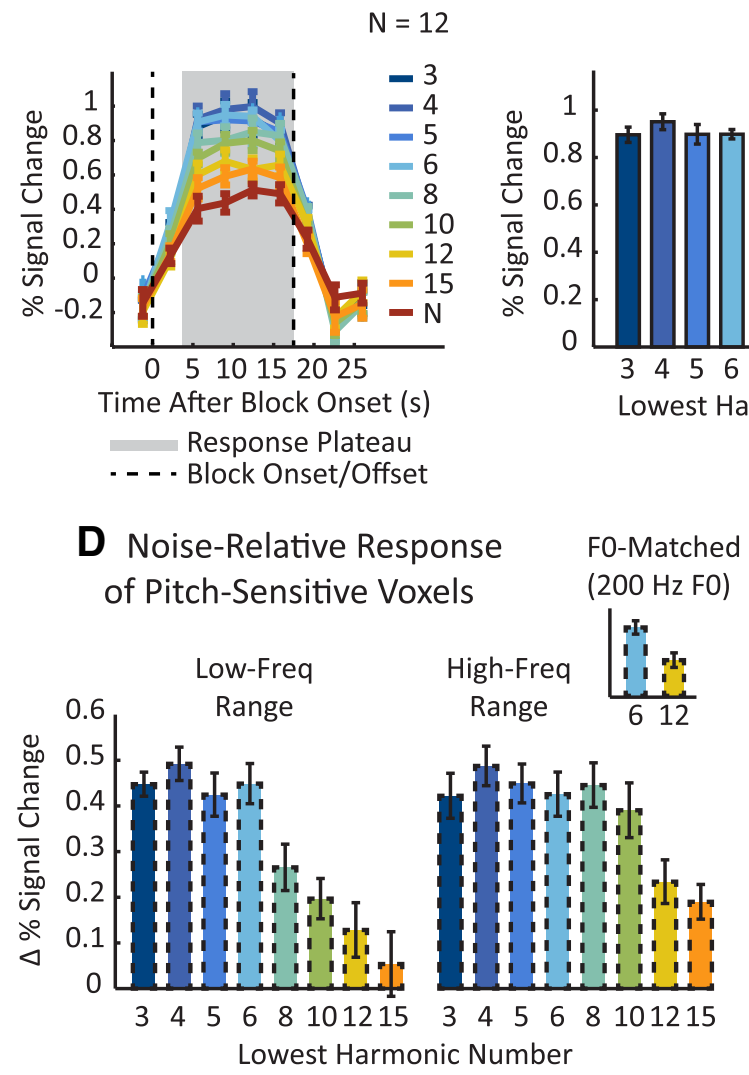

\section{Pitch Discrimination Thresholds}

$$
N=8
$$

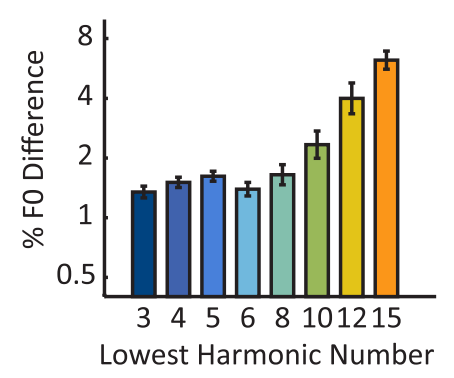


A Standard Anatomical ROIs

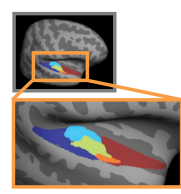

Right

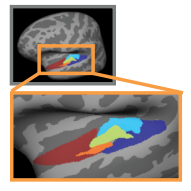

Left

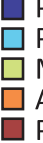

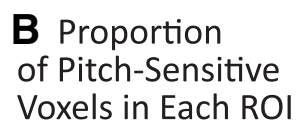

B Proportion Voxels in Each ROI
Planum Temporale

Posteromedial HG

Middle HG (TE1.0)

Anterolateral HG (TE1.2)

Planum Polare

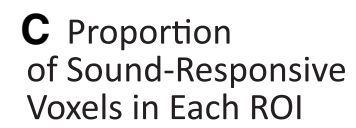

\section{Posterior-to-Anterior Anatomical ROIs}

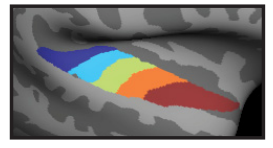

Right

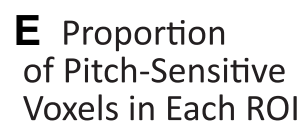

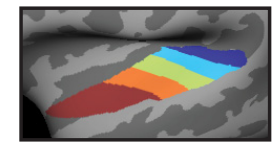

Left

\section{F Proportion of Sound-Responsive Voxels in Each ROI}

$\mathrm{N}=12$

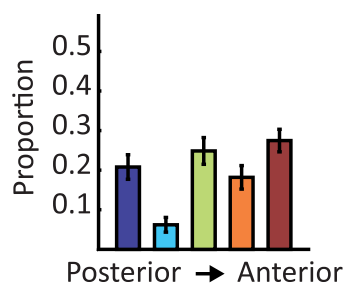

$\frac{\text { Pitch-Sens }}{\text { Sound-Resp }}$

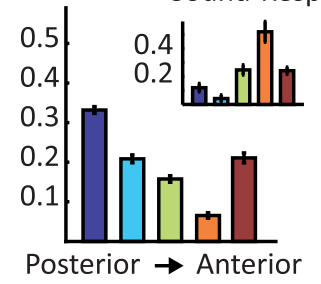

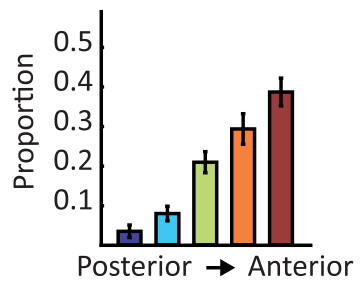

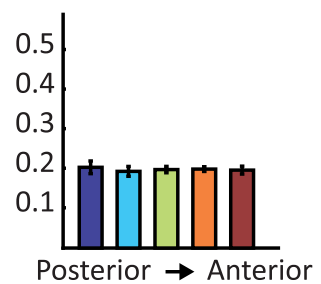

Figure 4. Anatomical distribution of pitch responses across auditory cortex. $A$, Standard anatomical ROIs displayed on an inflated average brain. $B$, Proportion of pitch-sensitive voxels in the superior temporal plane falling within each ROI. Pitch-sensitive voxels were identified by contrasting harmonic and noise conditions (see Fig. 5 for resolvability effects). C, Proportion of all significant sound-responsive voxels falling in each ROI. Sound-responsive voxels were defined by contrasting the response to all 18 harmonic and noise conditions with silence. Inset shows the proportion of sound-responsive voxels in each ROI that exhibited a pitch response and thus provides a measure of the density of pitch responses in each ROI. $D$, Novel set of anatomical ROls designed to run along the posterior-to-anterior axis of the superior temporal plane and to each include an equal number of sound-responsive voxels. $\boldsymbol{E}$, Proportion of all pitch-sensitive voxels falling within each posterior-to-anterior ROI. $\boldsymbol{F}$, Proportion of all significant sound-responsive voxels falling in each posterior-to-anterior ROI. Error bars indicate within-subject SEM.

tively). The exact "cutoff" point in the fMRI response varied slightly across the two frequency ranges, producing a significant interaction between harmonic number and frequency range $\left(F_{(7,11)}=3.16, p<0.01\right)$, with responses to lower frequency stimuli showing a lower cutoff point. This difference could plausibly be due to broader log-frequency tuning in low-frequency regions of the cochlea (Glasberg and Moore, 1990). It cannot be explained purely by a response preference for higher F0s, because stimuli matched in F0 exhibited a preference for resolved harmonics. Two conditions in particular were matched in F0 (with a mean $\mathrm{F} 0$ of $200 \mathrm{~Hz}$ ), but contained resolved harmonics in the low-frequency case (harmonics 6-12) and exclusively unresolved harmonics in the high-frequency case (harmonics 12-24). As highlighted in the inset of Figure $3 D$, responses were approximately twice as large in the low-frequency, resolved condition compared with the high-frequency, unresolved condition $\left(t_{(11)}=3.25, p<0.01\right)$, implicating resolvability rather than F0. Consistent with this interpretation, pitch discrimination thresholds were also lower for the condition with resolved harmonics (Fig. $3 E$, inset; $t_{(7)}=4.02, p<0.01$ ).

\section{Part 2: Anatomical location of cortical pitch responses}

The results from our analyses thus far demonstrate that pitchsensitive brain regions exhibit an overall response preference for resolved harmonics, but do not address where in the brain these pitch responses are located. To answer this question, we subdivided the superior temporal plane into five standard anatomical regions (Fig. $4 A$ ) and measured the number of pitch-sensitive voxels that fell within each region (Fig. $4 B$; pitch-sensitive voxels were again defined by contrasting harmonic tones with spectrallymatched noise). Because the distribution of pitch responses was similar for the left and right hemispheres, we combined ROIs across hemispheres. As a baseline, we also measured the distribution of sound-responsive voxels (Fig. 4C) defined by contrasting all harmonic and noise conditions with silence.

The distribution of pitch-sensitive voxels across anatomical ROIs differed qualitatively from the distribution of soundresponsive voxels. For example, the anterolateral HG ROI accounted for only $7 \%$ of sound-responsive voxels due to its small size, but comprised $18 \%$ of all pitch-sensitive voxels, consistent with prior studies that have reported pitch responses in anterolateral HG (Patterson et al., 2002; Penagos et al., 2004). When expressed as a fraction of the sound-responsive voxels they contain (Fig. 4C, inset), the three most anterior regions (middle $\mathrm{HG}$, anterolateral $\mathrm{HG}$, and planum polare) exhibited a higher proportion of pitch-sensitive voxels than did the two posterior regions (planum temporale and posteromedial HG). There was a highly significant main effect of region $\left(F_{(4,11)}=19.86, p<0.001\right)$ and all six direct contrasts between the three most anterior ROIs and the two most posterior ROIs were significant $\left(t_{(11)}>2.98\right.$ and $p<0.05)$. However, we nonetheless observed a substantial number of pitch-sensitive voxels in the planum temporale $(21 \%$ of all pitch-sensitive voxels were located there). This result revealed a limitation of the anatomical parcels used in this analysis: although the planum temporale is mostly posterior to the other anatomical ROIs, it includes a small anterior section that borders anterolateral $\mathrm{HG}$, which on inspection appeared to be the site of most of the pitch responses within the ROI.

To more directly test whether pitch responses are biased toward anterior regions of auditory cortex, we performed an analogous analysis with a new set of ROIs that were designed to run along the posterior-to-anterior axis of the superior temporal plane and to each include an equal number of sound-responsive voxels (Fig. $4 D$ ). Using these posterior-to-anterior ROIs, we observed a clear monotonic gradient, with a higher density of pitch responses in more anterior regions (Fig. $4 E$ ). In contrast, the 


\section{Response of Pitch-Sensitive Voxels in Different Anatomical ROls}

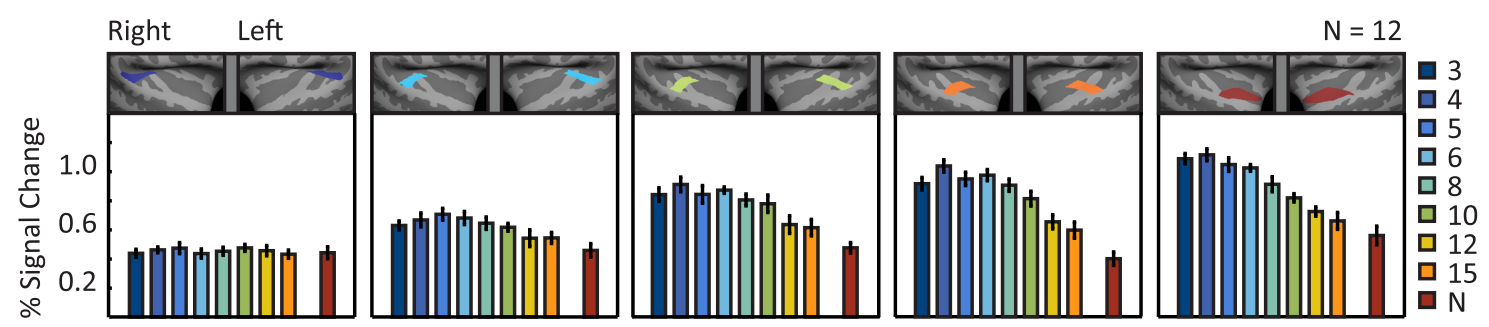

Figure 5. Anatomical distribution of resolvability effects across auditory cortex. The effect of resolvability and pitch on the response of pitch-sensitive voxels within each of five different anatomical ROls designed to run along the posterior-to-anterior axis of the superior temporal plane (same as in Fig. 4D). Responses were averaged across the two frequency ranges of our main parametric resolvability manipulation. Pitch-sensitive voxels were defined in independent data as the $10 \%$ of voxels in each ROI with the most significant response to harmonic tones compared with noise and thus are not guaranteed to exhibit a pitch response in independent data (as demonstrated by the most posterior region, which did not exhibit any replicable pitch responses). Error bars indicate one within-subject SEM.

baseline distribution of sound-responsive voxels was flat across the five ROIs, as intended (Fig. $4 F$ ).

\section{Anatomical distribution of resolvability effects across auditory cortex}

The fact that pitch responses are not distributed uniformly across different regions of auditory cortex raises the question of whether sensitivity to harmonic resolvability is also distributed nonuniformly. To address this issue, we measured the response of pitch-sensitive voxels from each of the five posterior-to-anterior ROIs to each harmonic and noise condition, collapsing across stimulus frequency range (Fig. 5). Consistent with our prior analysis, pitch responses were substantially more robust in the more anterior regions of the superior temporal plane, leading to a significant condition $\times$ ROI interaction $\left(F_{(32,11)}=9.69, p<0.001\right)$. Notably, however, every ROI with a large pitch response also exhibited a substantial response preference for low-numbered resolved harmonics (there was a significant main effect of harmonic number in the 4 most anterior ROIs: $F_{(7,1)}>2.1$ and $p<$ $0.05)$, suggesting that resolved harmonics drive pitch responses throughout auditory cortex.

\section{Consistency of pitch responses across subjects}

Our results show that pitch responses are primarily driven by resolved harmonics and are primarily limited to the anterior superior temporal plane. To test whether pitch responses are consistently observed in this anterior region across subjects, we identified pitch-sensitive voxels in individual subjects using our "efficient pitch localizer" (contrasting resolved harmonics with noise; see Materials and Methods) and then performed a $t$ test across data from the seven or eight independent runs from the main parametric experiment, comparing responses to resolved harmonic conditions (lowest harmonic less than the eighth) with responses to frequency-matched noise. In all 12 subjects tested, responses to resolved harmonics were significantly greater than those to noise $(p<0.05$ for 12 subjects using a one-tailed test). This result demonstrates that pitch-sensitive brain regions are a consistent feature of cortical organization and can be reliably identified in individual subjects by our localizer.

Part 3: The tonotopic location of pitch-sensitive brain regions To better characterize the anatomical variation in pitch responses found in the first two parts of our study, we explored the relationship between pitch sensitivity and the tonotopic map of auditory cortex. Tonotopy is perhaps the best known and most uncontroversial functional organizational principle in auditory cortex, and tonotopic maps are consistently lo- cated and oriented relative to macroanatomical landmarks (Talavage et al., 2004; Humphries et al., 2010; Da Costa et al., 2011; Moerel et al., 2012). In addition, primate neurophysiology studies have suggested that neurons tuned to individual pitches are clustered in the low-frequency portion of the tonotopic map (Bendor and Wang, 2005, 2010), so we sought to test whether a similar organization is present in humans (albeit using a very different neural measure).

Tonotopy was assessed as in prior studies by measuring responses to pure tones spanning 6 different frequency ranges (center frequencies of each range were $0.2,0.4,0.8,1.6,3.2$, and 6.4 $\mathrm{kHz}$ ) and pitch-sensitive voxels were identified by comparing responses to resolved harmonics (conditions including the eighth harmonic or lower) with frequency-matched noise. The latter choice followed from our earlier analysis, in which we found that resolved harmonics produced the most robust pitch responses and are thus best suited to localize them. Figure $6 \mathrm{~A}$ shows a whole-brain map, plotting the proportion of pitch responses at each point on the cortical surface. Figure $6 B$ shows a group tonotopy map for the same subjects with an outline of the pitchsensitive voxels from Figure $6 \mathrm{~A}$ overlaid. The map of pitchsensitivity replicated our anatomical analyses from Part 1, with pitch-sensitive voxels localized to the anterior half of superior temporal plane. Tonotopy maps exhibited a "V-shaped" highlow-high gradient oriented approximately perpendicular to HG, which is consistent with previous reports (Talavage et al., 2004; Woods et al., 2009; Humphries et al., 2010; Da Costa et al., 2011; Moerel et al., 2012; Baumann et al., 2013). Comparing pitch and tonotopy maps directly revealed a clear relationship between maps: pitch responses overlapped with low- but not highfrequency regions of primary auditory cortex.

To test directly whether pitch sensitivity is colocalized to regions selective for low frequencies, we identified voxels that responded more to either the three lowest or the three highest pure-tone conditions in each individual subject and measured their response to the resolved harmonic (conditions with a lowest harmonic below the eighth) and noise conditions for each of the two frequency ranges tested (Fig. 6C). Unsurprisingly, lowfrequency voxels exhibited a larger response for the lowerfrequency conditions (main effect of frequency: $F_{(1,11)}=88.82$, $p<0.001)$. Consistent with our whole-brain analysis, they also responded preferentially to harmonic tones compared with noise (main effect of stimulus type: $F_{(1,11)}=108.19, p<0.001$ ). In contrast, high-frequency voxels exhibited the expected larger response for the higher-frequency sounds, but responded preferentially to the noise conditions $\left(F_{(1,11)}=10.72, p<0.01\right)$. Together, these results indicate a strong low-frequency bias for 


\section{A Pitch-Sensitive Voxels}

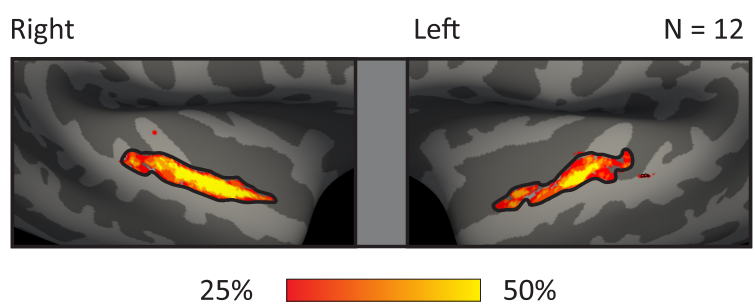

$\%$ of Subjects with a Pitch Response at Each Voxel

Pitch Response: Resolved Harmonics > Noise

\section{B Best-Frequency}

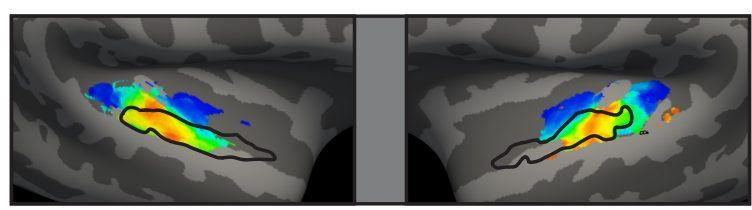

$0.2 \mathrm{kHz}$ $6.4 \mathrm{kHz}$

Frequency of Maximum Response

Outline of Pitch-Sensitive Voxels

D Frequency-Selectivity

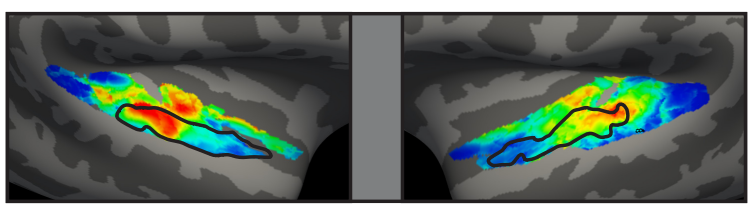

0.25

0.75

STD(Freq Response)/Mean(Freq Response)

Outline of Pitch-Sensitive Voxels

\section{Response of Low/High Frequency Voxels Identified Within Individual Subjects}

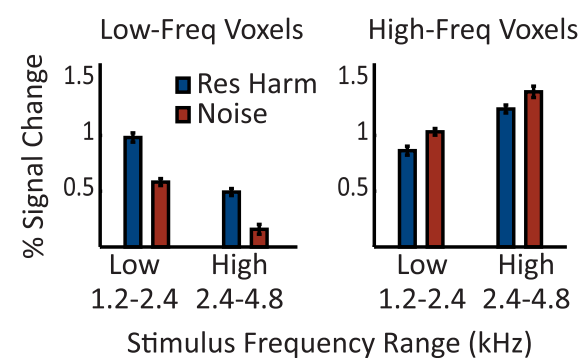

E Frequency-Selectivity of Pitch Voxels
Identified Within Individual Subjects

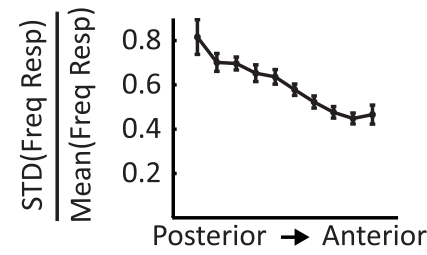

Anatomical Position of Pitch Voxels

Figure 6. The relationship among pitch responses, tonotopy, and frequency selectivity. $A$, Summary map indicating the percentage of subjects who exhibited a pitch response at each surface voxel. Pitch responses were identified with a contrast of resolved harmonics versus noise. $\boldsymbol{B}$, Best-frequency map showing the preferred frequency of each surface voxel averaged across subjects. An outline of the pitch-sensitive voxels from $A$ is overlaid for comparison. The preferred frequency of each voxel was defined in each subject as the pure-tone condition that produced the maximum response; these preferred frequencies were then averaged across subjects. The 6 pure-tone conditions correspond approximately to the colors red ( $0.2 \mathrm{kHz}$ center frequency), orange $(0.4 \mathrm{kHz})$, yellow-green $(0.8 \mathrm{kHz})$, blue-green $(1.6 \mathrm{kHz})$, light blue $(3.2 \mathrm{kHz})$, and dark blue $(6.4 \mathrm{kHz})$. C, The response of low-frequency-preferring (left) and high-frequency-preferring (right) voxels, defined within each subject individually, to low- and high-frequency-resolved harmonics (blue) and noise (red). Low- and high-frequency voxels were identified with a low versus high contrast ( $0.2+$ $0.4+0.8 \mathrm{kHz}$ vs $1.6+3.2+6.4 \mathrm{kHz}$ ). $\boldsymbol{D}$, Map of frequency selectivity indicating the degree to which a voxel responded differently across the six pure-tone conditions, overlaid with an outline of pitch-sensitive voxels. Frequency selectivity was calculated for each voxel as the standard deviation of the response to the six pure-tone conditions divided by the mean response (selectivity was computed within individual subjects and then averaged across participants to arrive at a group map). $\boldsymbol{E}$, Frequency selectivity of pitch-sensitive voxels plotted as a function of their posterior-toanterior position (each bar plots selectivity averaged across $10 \%$ of all pitch-sensitive voxels). Selectivity was calculated separately for each voxel in individual subjects and was then pooled across voxels with a similar posterior-to-anterior position. Error bars indicate one within-subject SEM.

pitch sensitivity. It is important to note that this relationship between pitch responses and frequency responses is not a trivial consequence of the frequency composition of the pitch localizer, because the stimuli used to identify pitch responses had their spectral energy at relatively high frequencies (the two frequency ranges spanned by the pitch localizer stimuli were centered on $1.2-2.4$ and $2.4-4.8 \mathrm{kHz}$ ).

Pitch-sensitive voxels also extended into more anterior regions of nonprimary auditory cortex with less selective frequency tuning. This drop in frequency selectivity is evident in Figure $6 D$, which plots a whole-brain map of frequency selectivity (standard deviation of responses to different frequencies divided by the mean) overlaid with the region in which pitch responses occur. To quantify this relationship more directly, we identified pitch-sensitive voxels in individual subjects and measured each voxel's degree of frequency tuning (again, defined as the standard deviation of the response to the different frequencies divided by the mean). We then averaged this selectivity measure across voxels with a similar posterior-toanterior position (Fig. 6E; see Materials and Methods for details). Consistent with the whole-brain analyses, we observed a monotonic gradient of frequency selectivity, with less selective responses in more anterior pitch voxels (main effect of position: $\left.F_{(9,11)}=10.3, p<0.001\right)$. Figure 7 shows maps of pitch responses, best frequency, and frequency selectivity in four individual subjects who participated in a follow-up scan 
A Pitch-Sensitive Voxels

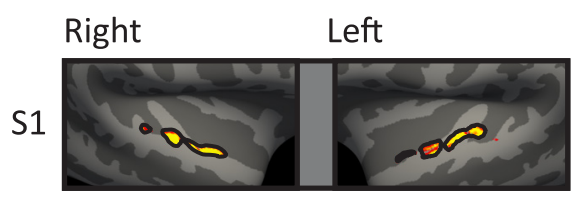

S2

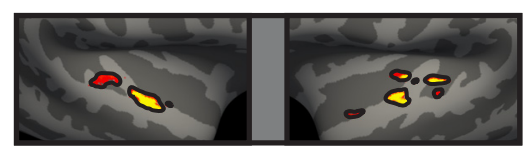

S3

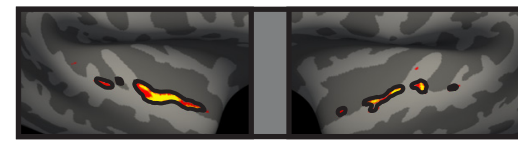

S4

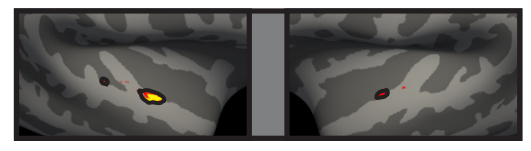

$10^{-3}$ $10^{-6}$

Significance of Pitch Response

Pitch Response: Res Harm > Noise

\section{B Best-Frequency}
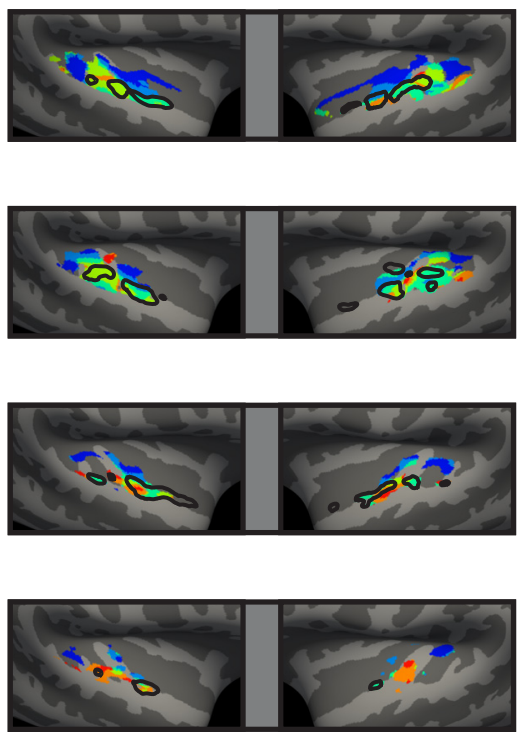

$0.2 \mathrm{kHz}$

$6.4 \mathrm{kHz}$

Freq of Max Response

- Pitch-Sensitive Voxels

\section{Frequency-Selectivity}
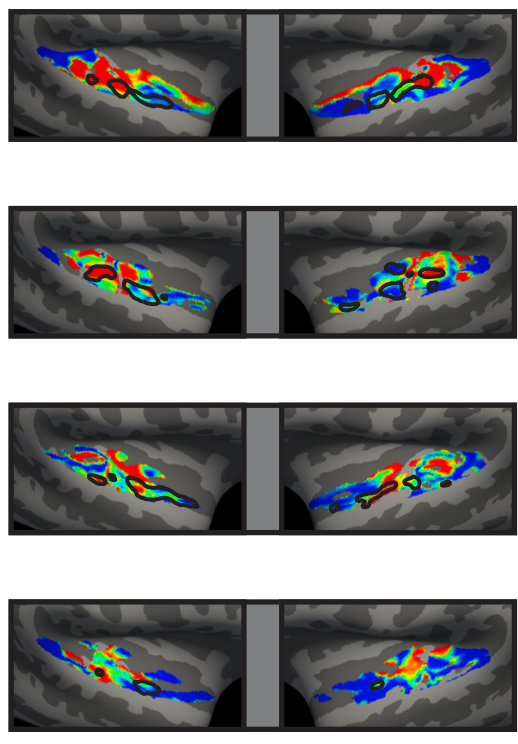

0.25

0.75

STD(Freq Resp)/Mean(Freq Resp)

- Pitch-Sensitive Voxels

Figure 7. Maps of pitch responses, tonotopy, and frequency selectivity in individual subjects. $A$, Pitch-sensitive voxels in four individual participants who participated in a follow-up scan (to more robustly measure pitch sensitivity and tonotopy). $\boldsymbol{B}$, Best-frequency maps for each participant with an outline of pitch-sensitive voxels from $\boldsymbol{A}$ overlaid. The best frequency of each voxel was defined as the frequency of the pure-tone condition that produced the maximum response. The 6 pure-tone conditions correspond approximately to the colors red $(0.2 \mathrm{kHz})$, orange $(0.4 \mathrm{kHz})$, yellow-green $(0.8 \mathrm{kHz})$, blue-green $(1.6 \mathrm{kHz})$, light blue $(3.2 \mathrm{kHz})$, and dark blue $(6.4 \mathrm{kHz})$. C, Maps of frequency selectivity for each individual participant with an outline of pitch-sensitive voxels overlaid. Frequency selectivity was defined as in Figure $6 C$.

(to ensure sufficient data to measure pitch and tonotopy responses robustly in their individual brains). As is evident from inspection, all four subjects exhibited the trends illustrated in our group analyses.

\section{Control experiment: Are resolvability effects dependent on frequency variation?}

Figure $8 A$ shows cochleograms for several example stimuli from the main experiment and illustrates the note-to-note frequency variation across the $6-12$ notes making up each $2 \mathrm{~s}$ stimulus. This variation was a design choice motivated by pilot experiments showing that such variation enhances the overall cortical response, with concomitant increases in the power to detect response differences across conditions. However, this variation also introduces a potential confound related to frequency adaptation: notes with spectrally resolved harmonics tend to have less frequency overlap with other notes in the sequence compared to notes without resolved harmonics, which could in principle lead to higher responses for resolved harmonics by reducing note-to-note frequency adaptation.

We addressed this potential confound with several sets of control conditions. We first tested whether neural resolvability effects persist for stimuli without any frequency variation by measuring the response of pitch-sensitive voxels to stimuli composed of a single, repeated note (Fig. $8 B$, top row). Pitch-sensitive voxels were defined by contrasting resolved harmonic tones with noise using stimuli from the main experiment. This analysis revealed a clear response preference for resolved harmonics compared with both unresolved harmonics $\left(t_{(7)}=2.95, p<0.05\right)$ and noise $\left(t_{(7)}=4.91, p<0.01\right.$; Fig. $\left.8 C\right)$, demonstrating that resolvability effects are not dependent on frequency variation and are not simply a byproduct of frequency adaptation.

The presence of a resolvability effect for single repeated notes does not rule out the possibility that frequency adaptation could have contributed to the resolvability effects observed in the main experiment. To address this question, we measured responses to stimuli with two alternating notes and manipulated the spectral overlap between the two notes directly (Fig. $8 B$, bottom two rows). In one condition (two-notes overlapping), the two notes had overlapping frequency ranges that were designed to minimize overlap for resolved harmonics while maximizing overlap for unresolved harmonics. In the other condition (two-notes nonoverlapping), the two notes occupied distinct frequency regions and therefore never overlapped. If differences in note-tonote frequency overlap due to resolvability affect the cortical response, there should be a larger response difference between resolved and unresolved harmonics for the notes with overlapping frequency ranges.

Contrary to this prediction, pitch-sensitive voxels responded similarly to the overlapping and nonoverlapping conditions (Fig. $8 C$ ). There was a clear response preference for resolved harmonics compared with either unresolved harmonics or noise in both cases $\left(t_{(7)}>4.57, p<0.01\right.$ for all direct comparisons), with no main effect of overlap $\left(F_{(1,7)}=1.10, p=0.33\right)$ and no interaction between overlap and note type $\left(F_{(2,7)}=0.30, p=0.75\right)$. These results demonstrate that differential frequency adaptation cannot explain the resolvability effect of our main experiment and likely does not contribute to it to any substantial degree. 


\section{A Example Stimuli from Main Experiment}

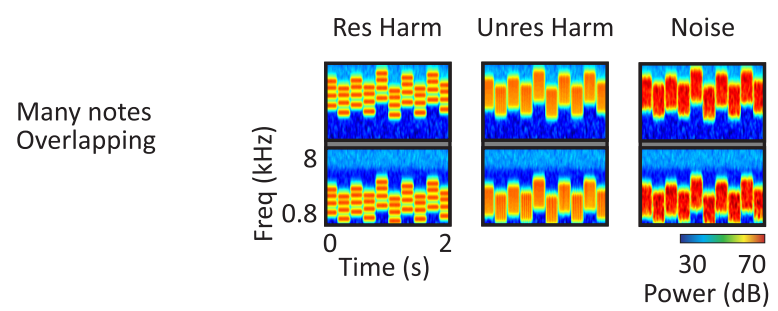

B Example Stimuli from Control Experiment

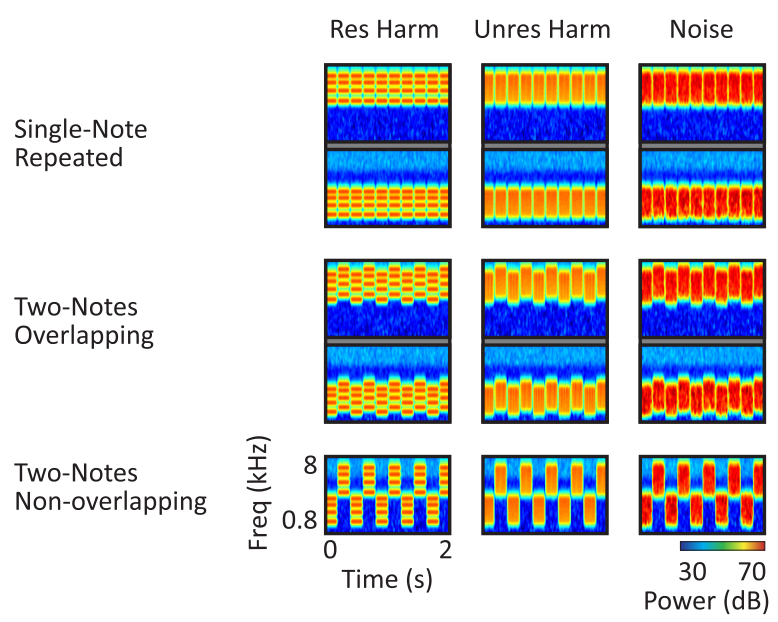

C Response of Pitch-Sensitive Voxels

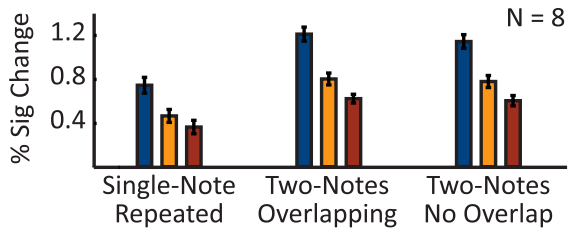

D Response of High-Frequency Voxels

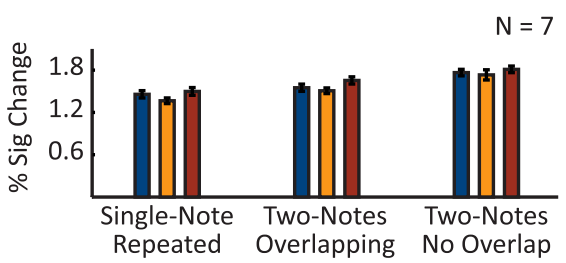

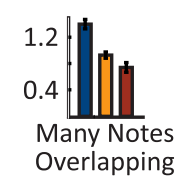

QRes Harm -Unres Harm $\square$ Noise

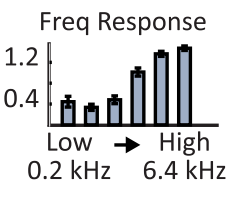

Figure 8. Effect of frequency variation on cortical responses to resolved and unresolved harmonics. A, Cochleograms for example resolved (left, harmonics 3-6), unresolved (middle, harmonics 15-30), and noise (right) stimuli from the main experiment. Each stimulus was composed of several different notes with overlapping frequency ranges. Low- (bottom) and high- (top) frequency examples are shown for each note type. Cochleograms were computed as in Figure $2 B$. $\boldsymbol{B}$, Cochleograms illustrating the $3 \times 3$ factorial design used in the control experiment. Stimuli were composed of a single repeated note (top row), two alternating notes with overlapping frequency ranges (middle row), or alternating notes with nonoverlapping frequency ranges (bottom row). Each note was composed of resolved harmonics (left column, harmonics 3-6), unresolved harmonics (middle column, harmonics 15-30), or noise (right column). All conditions spanned a frequency range similar to that of the stimuli in the main experiment. $C$, Response of pitch-sensitive voxels to the nine conditions of the control experiment. Pitch-sensitive voxels were identified by contrasting resolved harmonics with noise using the stimuli from the main experiment (illustrated in $\boldsymbol{A}$ ). The inset shows responses to the analogous resolved harmonics, unresolved harmonics, and noise conditions from the main experiment (measured in data independent of the localizer). $\boldsymbol{D}$, Response of high-frequencypreferring voxels to the nine conditions of the control experiment. High-frequency voxels were identified by contrasting high- $(1.6,3.2,6.4 \mathrm{kHz})$ and low- $(0.2,0.4,0.8 \mathrm{kHz})$ frequency pure tones. The inset shows responses to the six pure-tone conditions used to localize the region (measured in data independent of the localizer). Error bars indicate one within-subject SEM.
As expected, we also observed a larger overall response for both two-note conditions relative to the conditions with a single repeated note $\left(t_{(7)}>5.80, p<0.001\right.$ for both comparisons; main effect of the three types of note variation, $F_{(2,7)}=37.63$, $p<$ $0.001)$. Moreover, the effect of note variation increased in more anterior pitch regions: pitch-sensitive voxels in the middle ROI of the posterior-to-anterior analysis (the most posterior ROI that exhibited a substantial pitch response; Figs. $4 D-F$ ) responded $28 \%$ more to the two-note conditions than to the single-note conditions, whereas pitch-sensitive voxels in the most anterior ROI responded $80 \%$ more to the two-note conditions. The normalized difference between two-note and single-note conditions ([two-note - single-note]/[two-note + single-note $]$ ) was significantly larger in the anterior region than the middle region $\left(t_{(7)}=\right.$ $3.63, p<0.01)$. This result is consistent with a number of studies reporting greater responses to stimuli with variable pitches/frequencies in more anterior regions of auditory cortex (Patterson et al., 2002; Warren and Griffiths, 2003; Puschmann et al., 2010). However, there was no interaction between note variation and note type in either the middle $\left(F_{(4,7)}=1.23, p=0.32\right)$ or the most anterior ROI $\left(F_{(4,7)}=1.44 ; p=0.25\right)$, demonstrating that resolvability effects are observed regardless of pitch/frequency variation.

To further explore any potential effects of frequency adaptation, we examined the response of tonotopic regions, because these might be expected to show the largest effects of frequency adaptation (Fig. $8 D$ ). We focused on voxels preferring high frequencies both because they are distinct from pitch-sensitive voxels (as demonstrated in the main experiment; Figs. 6, 7) and because all of the harmonic and noise stimuli had their power at relatively high frequencies $(>1 \mathrm{kHz})$. As in the main experiment, the response of high-frequency-preferring voxels to resolved, unresolved, and noise stimuli was similar. There was no significant main effect of note type $\left(F_{(2,6)}=2.45, p=0.13\right)$ and no interaction between note type and note variation $\left(F_{(4,6)}=0.36, p=\right.$ $0.83)$. These results suggest that differences in spectral overlap due to resolvability have little effect on cortical response magnitudes, even in regions in which responses are strongly modulated by frequency and even when stimuli are designed explicitly to maximize differences in spectral overlap. One explanation for the lack of a resolvability-linked adaptation effect is that cortical frequency tuning could be relatively coarse, such that the subtle note-to-note spectral differences present in our stimuli are insufficient to alter frequency adaptation. Consistent with this notion, we observed an overall difference in the response of high-frequency-preferring voxels to the three types of note variation $\left(F_{(2,6)}=58.87, p<0.001\right)$, with larger responses for stimuli with fully nonoverlapping frequency ranges, as might be expected from frequency adaptation at a relatively coarse spectral scale.

\section{Discussion}

Our study demonstrates two key properties of responses to pitch in the human brain and helps to reconcile apparent inconsistencies in the prior literature on pitch processing. First, pitch responses throughout cortex are predominantly driven by the presence of resolved harmonics, consistent with the importance of spectral cues in human pitch perception (Houtsma and Smurzinski, 1990; Shackleton and Carlyon, 1994). Responses to unresolved harmonics (with purely temporal pitch cues) were much weaker than those to resolved harmonics. Moreover, the response of pitch-sensitive regions to a parametric manipulation of resolvability closely mirrored behavioral discrimination thresholds for the same stimuli. Second, pitch responses exhibit a 
consistent and stereotyped anatomy, mostly occurring in the anterior half of auditory cortex, localized to low-frequency regions of primary auditory cortex and less frequency-selective regions of nonprimary auditory cortex. In the remaining sections, we show how these two findings fit with, and resolve apparent inconsistencies among, prior studies of pitch processing in the brain.

\section{Harmonic resolvability}

Although many neuroimaging studies have investigated pitch responses (Griffiths et al., 1998; Gutschalk et al., 2002, 2004; Patterson et al., 2002; Krumbholz et al., 2003; Penagos et al., 2004; Hall et al., 2005, 2006; Chait et al., 2006), the anatomy and response properties of pitch-sensitive regions have remained controversial (Hall and Plack, 2007, 2009; Griffiths et al., 2010; Puschmann et al., 2010; Barker et al., 2011, 2012; Griffiths and Hall, 2012; Sedley et al., 2012). One potential reason for this lack of consensus is that most studies have focused on pitch stimuli with unresolved harmonics (but see Schönwiesner and Zatorre, 2008; Hall and Plack, 2009; Steinmann and Gutschalk, 2012). Such stimuli produce a weak pitch percept, and we found them to produce a weak neural response throughout auditory cortex compared with stimuli with resolved harmonics (Fig. 3). Our results thus help to explain why many recent studies have failed to observe robust pitch responses using unresolved pitch stimuli (Hall and Plack, 2007; Garcia et al., 2010; Barker et al., 2011, 2012). Earlier reports of localized responses in anterolateral HG to temporal pitch cues can be reconciled with our findings by the fact that many such studies used stimuli containing some resolved harmonics, similar to those that produced a large neural response in our study (Griffiths et al., 1998; Gutschalk et al., 2002; Patterson et al., 2002; Hall et al., 2005; Barrett and Hall, 2006). For example, the pitch stimuli used by Patterson et al. (2002) included harmonics as low as the fifth depending on the note (F0s ranged from 50 to $110 \mathrm{~Hz}$ and were band-pass filtered between 500 and $4000 \mathrm{~Hz}$ ). Therefore, the large majority of prior results are consistent with the response profile of Figure $3 B$, in which pitch responses are robust when stimuli contain harmonics below the eighth, for which pitch perception is most acute (see Hall and Plack, 2009, for a possible exception).

Our results are also consistent with those of Penagos et al. (2004), who reported a greater response to a resolved pitch stimulus than to three unresolved pitch stimuli in a region near anterolateral HG, using stimuli with note-to-note frequency variation. Our results extend their findings in three respects. First, we show that pitch-sensitive regions throughout cortex respond more to resolved than unresolved harmonics by as much as a factor of 2 or 3 (relative to noise), even though the contrast we used to identify pitch regions (harmonic tones $>$ noise) was independent of resolvability. Second, we show that the response profile of these regions closely tracks the effect of resolvability on pitch perception, with a relatively high, constant response to harmonics below the eighth and monotonic decrease for higher harmonics. Third, we demonstrate that cortical resolvability effects do not depend on note-to-note frequency variation and are thus not a byproduct of adaptation to frequency.

Finally, although our findings indicate the importance of resolved spectral cues, we nonetheless observed a small but significant response for unresolved harmonics relative to noise, which is consistent with some role for temporal pitch cues (Patterson et al., 2002; Barker et al., 2011). Because our harmonic complexes were simply combinations of pure tones and because we observed similar pitch responses for stimuli with and without frequency variation, our results are not susceptible to the concerns regarding spectrotemporal modulation that have been raised for “iterated-ripple-noise" stimuli (Barker et al., 2012; Bendor, 2012) (although our results do not rule out the possibility that pitchsensitive regions may also respond to slow spectrotemporal modulations as suggested by Barker et al., 2013). In addition, the responses we observed to unresolved harmonics are unlikely to be due to cochlear distortion because we used noise to mask distortion products. Our findings are thus broadly consistent with models of pitch perception that rely on both spectral and temporal pitch cues, and with primate neurophysiology studies reporting sensitivity to both kinds of cues in neurons of primary auditory cortex (Bendor et al., 2012; Wang and Walker, 2012; Fishman et al., 2013). Our findings are also consistent with the more general hypothesis that pitch-sensitive regions respond in proportion to pitch salience, even for acoustic manipulations that do not alter harmonic resolvability (Barker et al., 2013). This question could be addressed in future research by identifying pitch-sensitive regions using resolved harmonics (which have high pitch salience) and then measuring the influence of other manipulations of pitch salience on the response of the identified regions.

\section{Anatomical and tonotopic location of pitch responses}

Our results demonstrate that pitch responses exhibit a consistent and stereotyped anatomy, being located primarily in anterior regions of auditory cortex and in specific regions of the tonotopic map. These findings contrast with studies reporting more distributed responses throughout cortex, a discrepancy that we argue is in part due to the use of unresolved pitch stimuli in many prior studies (Hall and Plack, 2007; Garcia et al., 2010; Barker et al., 2011, 2012). Many of these same studies have also reported a focal point of pitch sensitivity in the planum temporale, a relatively posterior anatomical region. We also observed pitch responses in the planum temporale, but in our data, these responses were localized to the anterior tail of this region, bordering anterolateral HG. We confirmed this observation using a novel set of ROIs designed to run along the posterior-to-anterior axis of auditory cortex. These ROIs revealed that the density of pitch responses increases monotonically from posterior to anterior auditory cortex.

The responses that we observed in anterolateral $\mathrm{HG}$ replicate a number of previous reports (Gutschalk et al., 2002; Patterson et al., 2002; Penagos et al., 2004; Hall et al., 2005; Puschmann et al., 2010), but the responses we observed in more anterior regions of planum polare have less precedent (but see Barrett and Hall, 2006; Barker et al., 2011). One plausible explanation is that responses in more anterior pitch regions may have been enhanced by the pitch variation in our stimuli, consistent with prior reports of responses in HG for fixed pitch stimuli (Gutschalk et al., 2002; Patterson et al., 2002; Hall et al., 2005; Hall and Plack, 2009; Puschmann et al., 2010) but anterior to HG for variable pitch stimuli (Patterson et al., 2002; Warren and Griffiths, 2003; Puschmann et al., 2010). This possibility is supported directly by our finding that stimuli with pitch and frequency variation produce a larger overall response, with a larger response increment in more anterior pitch regions.

Our finding that pitch responses occur in low-frequency regions of the tonotopic map suggests a link with primate neurophysiology studies that have reported pitch-tuned neurons clustered in a low-frequency region near the border of A1 and R (Bendor and Wang, 2005, 2006, 2010; Wang and Walker, 2012). 
The pitch responses observed here extended beyond this lowfrequency region into more anterior and less frequency-selective regions of auditory cortex, but the possible homology is nonetheless suggestive (see also Hall et al., 2006). Because our pitch stimuli included low-frequency masking noise, this relationship between pitch and frequency sensitivity is unlikely to be explained simply by responses to low-frequency distortion products. We note that the relationship between pitch and frequency sensitivity bears an intriguing resemblance to the relationship between pitch information and frequency in speech. Harmonic speech sounds generally have their power concentrated at relatively low frequencies, whereas unvoiced speech sounds, such as fricatives, typically have a high-pass spectrum (due to air being forced through a narrow constriction at the front of the vocal tract; Stevens, 2000). One speculative possibility is that pitch analysis is frequency dependent in a way that is adapted to the statistics of natural sounds.

Finally, another notable difference between our study and most prior neuroimaging studies of pitch is that we used functional localizer contrasts to identify pitch-sensitive ROIs within individual subjects. This distinction is potentially important because functionally defined brain regions never occur in exactly the same anatomical location across individuals. Group analyses might therefore fail to identify regions that can nevertheless be easily identified in individual subjects with a simple functional contrast (e.g., resolved harmonics $>$ noise).

\section{Outstanding questions}

Our study indicates that pitch responses are a systematic feature of auditory cortical organization, but many important questions remain. Do neurons within pitch-sensitive regions exhibit tuning to F0 across changes in spectrum (Bendor and Wang, 2005; Walker et al., 2011)? Are pitch-sensitive regions selectively involved in processing pitch relative to other sound properties (Warren and Griffiths, 2003; Bendor and Wang, 2005, 2010; Lewis et al., 2009; Bizley and Walker, 2010; Walker et al., 2011)? What role do pitch-sensitive regions play in computing higherlevel pitch-dependent representations such as speech prosody (Meyer et al., 2004) and musically relevant interval and contour information (Dowling, 1978; Peretz et al., 1994; Zatorre et al., 2002; McDermott et al., 2008, 2010; Lee et al., 2011; Schindler et al., 2013; Tierney et al., 2013)? The methods developed here for identifying and measuring pitch-sensitive regions in individual subjects should provide a useful tool for answering these questions.

\section{Notes}

Supplemental material for this article is available at http://web.mit. edu/svnh/www/Resolvability/Stimuli.html. This material has not been peer reviewed.

\section{References}

Barker D, Plack CJ, Hall DA (2011) Human auditory cortical responses to pitch and to pitch strength. Neuroreport 22:111-115. CrossRef Medline

Barker D, Plack CJ, Hall DA (2012) Reexamining the evidence for a pitchsensitive region: a human fMRI study using iterated ripple noise. Cereb Cortex 22:745-753. CrossRef Medline

Barker D, Plack CJ, Hall DA (2013) Representations of pitch and slow modulation salience in auditory cortex. Front Syst Neurosci 7:62. CrossRef Medline

Barrett DJ, Hall DA (2006) Response preferences for "what" and "where" in human non-primary auditory cortex. Neuroimage 32:968-977. CrossRef Medline

Baumann S, Petkov CI, Griffiths TD (2013) A unified framework for the organization of auditory cortex. Front Syst Neurosci 7:1-8. CrossRef Medline

Bendor D (2012) Does a pitch center exist in auditory cortex? J Neurophysiol 107:743-746. CrossRef Medline

Bendor D, Wang X (2005) The neuronal representation of pitch in primate auditory cortex. Nature 436:1161-1165. CrossRef Medline

Bendor D, Wang X (2006) Cortical representations of pitch in monkeys and humans. Curr Opin Neurobiol 16:391-399. CrossRef Medline

Bendor D, Wang X (2010) The neuronal coding of periodicity in marmoset auditory cortex. J Neurophysiol 103:1809-1822. CrossRef Medline

Bendor D, Osmanski MS, Wang X (2012) Dual-pitch processing mechanisms in primate auditory cortex. J Neurosci 32:16149-16161. CrossRef Medline

Bernstein JG, Oxenham AJ (2005) An autocorrelation model with place dependence to account for the effect of harmonic number on fundamental frequency discrimination. J Acoust Soc Am 117:3816-3831. CrossRef Medline

Bizley JK, Walker KM (2010) Sensitivity and selectivity of neurons in auditory cortex to the pitch, timbre, and location of sounds. Neuroscientist 16:453-469. CrossRef Medline

Carlyon RP, Shackleton TM (1994) Comparing the fundamental frequencies of resolved and unresolved harmonics: evidence for two pitch mechanisms. J Acoust Soc Am 95:3541-3554. CrossRef

Chait M, Poeppel D, Simon JZ (2006) Neural response correlates of detection of monaurally and binaurally created pitches in humans. Cereb Cortex 16:835-848. CrossRef Medline

Da Costa S, van der Zwaag W, Marques JP, Frackowiak RS, Clarke S, Saenz M (2011) Human primary auditory cortex follows the shape of Heschl's gyrus. J Neurosci 31:14067-14075. CrossRef Medline

Desikan RS, Ségonne F, Fischl B, Quinn BT, Dickerson BC, Blacker D, Buckner RL, Dale AM, Maguire RP, Hyman BT, Albert MS, Killiany RJ (2006) An automated labeling system for subdividing the human cerebral cortex on MRI scans into gyral based regions of interest. Neuroimage 31:968 980. CrossRef Medline

Dowling WJ (1978) Scale and contour: two components of a theory of memory for melodies. Psychological Review 85:341-354. CrossRef

Ellis DPW (2009) Gammatone-like spectrograms. Available from: http:// www.ee.columbia.edu/ln/rosa/matlab/gammatonegram/. Accessed November 15, 2013.

Fastl H (1980) Pitch strength and masking patterns of low-pass noise. In: Psychophysical, physiological, and behavioral studies in hearing (Brink GVD, Bilsen F, eds), pp 334-339. Delft, The Netherlands: Delft UP.

Fischl B, Sereno MI, Dale AM (1999) Cortical surface-based analysis. II. Inflation, flattening, and a surface-based coordinate system. Neuroimage 9:195-207. CrossRef Medline

Fishman YI, Micheyl C, Steinschneider M (2013) Neural representation of complex tones in primary auditory cortex of the awake monkey. J Neurosci 33:10312-10323. CrossRef Medline

Garcia D, Hall DA, Plack CJ (2010) The effect of stimulus context on pitch representations in the human auditory cortex. Neuroimage 51:808-816. CrossRef Medline

Glasberg BR, Moore BCJ (1990) Derivation of auditory filter shapes from notched-noise data. Hearing Res 47:103-138. CrossRef Medline

Glasberg BR, Moore BC (2006) Prediction of absolute thresholds and equalloudness contours using a modified loudness model. J Acoust Soc Am 120:585-588. CrossRef Medline

Goldstein JL (1967) Auditory nonlinearity. J Acoust Soc Am 41:676-689. CrossRef Medline

Goldstein JL (1973) An optimum processor theory for the central formation of the pitch of complex tones. J Acoust Soc Am 54:1496-1516. CrossRef Medline

Greve DN, Fischl B (2009) Accurate and robust brain image alignment using boundary-based registration. Neuroimage 48:63-72. CrossRef Medline

Griffiths TD, Hall DA (2012) Mapping pitch representation in neural ensembles with fMRI. J Neurosci 32:13343-13347. CrossRef Medline

Griffiths TD, Büchel C, Frackowiak RS, Patterson RD (1998) Analysis of temporal structure in sound by the human brain. Nat Neurosci 1:422427. CrossRef Medline

Griffiths TD, Kumar S, Sedley W, Nourski KV, Kawasaki H, Oya H, Patterson RD, Brugge JF, Howard MA (2010) Direct recordings of pitch responses from human auditory cortex. Curr Biol 20:1128-1132. CrossRef Medline 
Gutschalk A, Patterson RD, Rupp A, Uppenkamp S, Scherg M (2002) Sustained magnetic fields reveal separate sites for sound level and temporal regularity in human auditory cortex. Neuroimage 15:207-216. CrossRef Medline

Gutschalk A, Patterson RD, Scherg M, Uppenkamp S, Rupp A (2004) Temporal dynamics of pitch in human auditory cortex. Neuroimage 22:755766. CrossRef Medline

Hall DA, Plack CJ (2007) The human 'pitch center' responds differently to iterated noise and Huggins pitch. Neuroreport 18:323-327. CrossRef Medline

Hall DA, Plack CJ (2009) Pitch processing sites in the human auditory brain. Cereb Cortex 19:576-585. CrossRef Medline

Hall DA, Haggard MP, Akeroyd MA, Palmer AR, Summerfield AQ, Elliott MR, Gurney EM, Bowtell RW (1999) "Sparse" temporal sampling in auditory fMRI. Hum Brain Mapp 7:213-223. CrossRef Medline

Hall DA, Barrett DJ, Akeroyd MA, Summerfield AQ (2005) Cortical representations of temporal structure in sound. J Neurophysiol 94:3181-3191. CrossRef Medline

Hall DA, Edmondson-Jones AM, Fridriksson J (2006) Periodicity and frequency coding in human auditory cortex. Eur J Neurosci 24:3601-3610. CrossRef Medline

Houtsma AJM, Smurzinski J (1990) Pitch identification and discrimination for complex tones with many harmonics. J Acoust Soc Am 87:304-310. CrossRef

Humphries C, Liebenthal E, Binder JR (2010) Tonotopic organization of human auditory cortex. Neuroimage 50:1202-1211. CrossRef Medline

Jenkinson M (1999) Measuring transformation error by RMS deviation: FMRIB Technical Report. Available from: http://fsl.fmrib.ox.ac.uk/ analysis/techrep/tr99mj1/tr99mj1.pdf. Accessed November 15, 2013.

Jenkinson M, Smith S (2001) A global optimisation method for robust affine registration of brain images. Medical Image Analysis 5:143-156. CrossRef Medline

Krumbholz K, Patterson RD, Seither-Preisler A, Lammertmann C, Lütkenhöner B (2003) Neuromagnetic evidence for a pitch processing center in Heschl's gyrus. Cereb Cortex 13:765-772. CrossRef Medline

Lee YS, Janata P, Frost C, Hanke M, Granger R (2011) Investigation of melodic contour processing in the brain using multivariate pattern-based fMRI. Neuroimage 57:293-300. CrossRef Medline

Levitt H (1971) Transformed up-down methods in psychoacoustics. J Acoust Soc Am 46:467-477. Medline

Lewis JW, Talkington WJ, Walker NA, Spirou GA, Jajosky A, Frum C, Brefczynski-Lewis JA (2009) Human cortical organization for processing vocalizations indicates representation of harmonic structure as a signal attribute. J Neurosci 29:2283-2296. CrossRef Medline

Licklider JCR (1954) "Periodicity" pitch and "place" pitch. J Acoust Soc Am 26:945. CrossRef

McDermott JH, Lehr AJ, Oxenham AJ (2008) Is relative pitch specific to pitch? Psychol Sci 19:1263-1271. CrossRef Medline

McDermott JH, Lehr AJ, Oxenham AJ (2010) Individual differences reveal the basis of consonance. Curr Biol 20:1035-1041. CrossRef Medline

Meddis R, Hewitt MJ (1991) Virtual pitch and phase sensitivity of a computer model of the auditory periphery. I: Pitch identification. J Acoust Soc Am 89:2866-2882. CrossRef

Meyer M, Steinhauer K, Alter K, Friederici AD, von Cramon DY (2004) Brain activity varies with modulation of dynamic pitch variance in sentence melody. Brain Lang 89:277-289. CrossRef Medline

Micheyl C, Keebler MV, Oxenham AJ (2010) Pitch perception for mixtures of spectrally overlapping harmonic complex tones. J Acoust Soc Am 128: 257-269. CrossRef Medline

Moerel M, de Martino F, Formisano E (2012) Processing of natural sounds in human auditory cortex: tonotopy, spectral tuning, and relation to voice sensitivity. J Neurosci 32:14205-14216. CrossRef Medline

Moore BC, Huss M, Vickers DA, Glasberg BR, Alcántara JI (2000) A test for the diagnosis of dead regions in the cochlea. Br J Audiol 34:205-224. CrossRef Medline

Morosan P, Rademacher J, Schleicher A, Amunts K, Schormann T, Zilles K (2001) Human primary auditory cortex: cytoarchitectonic subdivisions and mapping into a spatial reference system. Neuroimage 13:684-701. CrossRef Medline

Norman KA, Polyn SM, Detre GJ, Haxby JV (2006) Beyond mind-reading: multi-level pattern analysis of fMRI data. Trends Cogn Sci 10:424-430. CrossRef Medline
Oxenham AJ (2012) Pitch perception. J Neurosci 32:13335-13338. CrossRef Medline

Patterson RD, Robinson JH, McKeown D, Zhang C, Allerhand M (1992) Complex sounds and auditory images. In: Auditory physiology and perception: Proceedings of the 9th International Symposium on Hearing (Cazals Y, Demany L, Horner K, eds), pp 429-446. Oxford: Pergamon.

Patterson RD, Uppenkamp S, Johnsrude IS, Griffiths TD (2002) The processing of temporal pitch and melody information in auditory cortex. Neuron 36:767-776. CrossRef Medline

Peelle JE, Eason RJ, Schmitter S, Schwarzbauer C, Davis MH (2010) Evaluating an acoustically quiet EPI sequence for use in fMRI studies of speech and auditory processing. Neuroimage 52:1410-1419. CrossRef Medline

Penagos H, Melcher JR, Oxenham AJ (2004) A neural representation of pitch salience in nonprimary human auditory cortex revealed with functional magnetic resonance imaging. J Neurosci 24:6810-6815. CrossRef Medline

Peretz I, Kolinsky R, Tramo M, Labrecque R, Hublet C, Demeurisse G, Belleville S (1994) Functional dissociations following bilateral lesions of auditory cortex. Brain 117:1283-1301. CrossRef Medline

Plack CJ, Oxenham AJ, Fay RR, Popper AN (2005) Pitch: neural coding and perception New York: Springer.

Pressnitzer D, Patterson RD (2001) Distortion products and the perceived pitch of harmonic complex tones. In: Physiological and psychophysical bases of auditory function (Breebart DJ, Houtsma AJM, Kohlrausch A, Prijs VF, Schoonoven R, eds), pp 97-104. Maastricht: Shaker Publishing BV.

Puschmann S, Uppenkamp S, Kollmeier B, Thiel CM (2010) Dichotic pitch activates pitch processing centre in Heschl's gyrus. Neuroimage 49:16411649. CrossRef Medline

Schindler A, Herdener M, Bartels A (2013) Coding of melodic gestalt in human auditory cortex. Cereb Cortex 23:2987-2993. CrossRef Medline

Schmitter S, Diesch E, Amann M, Kroll A, Moayer M, Schad LR (2008) Silent echoplanar imaging for auditory fMRI. MAGMA 21:317-325. CrossRef Medline

Schönwiesner M, Zatorre RJ (2008) Depth electrode recordings show double dissociation between pitch processing in lateral Heschl's Gyrus and sound onset processing in medial Heschl's gyrus. Exp Brain Res 187:97105. CrossRef Medline

Schroeder M (1970) Synthesis of low-peak-factor signals and binary sequences with low autocorrelation. IEEE Transactions on Information Theory 16:85-89. CrossRef

Sedley W, Teki S, Kumar S, Overath T, Barnes GR, Griffiths TD (2012) Gamma band pitch responses in human auditory cortex measured with magnetoencephalography. Neuroimage 59:1904-1911. CrossRef Medline

Shackleton TM, Carlyon RP (1994) The role of resolved and unresolved harmonics in pitch perception and frequency modulation discrimination. J Acoust Soc Am 95:3529-3540. CrossRef Medline

Slaney M (1998) Auditory toolbox version 2. Interval Research Corporation Technical Report \#1998-010. Available from: https://engineering. purdue.edu/ malcolm/interval/1998-010/. Accessed November 15, 2013.

Small AM Jr, Daniloff RG (1967) Pitch of noise bands. J Acoust Soc Am 41:506-512. CrossRef Medline

Smith SM, Jenkinson M, Woolrich MW, Beckmann CF, Behrens TE, Johansen-Berg H, Bannister PR, De Luca M, Drobnjak I, Flitney DE, Niazy RK, Saunders J, Vickers J, Zhang Y, De Stefano N, Brady JM, Matthews PM (2004) Advances in functional and structural MR image analysis and implementation as FSL. Neuroimage 23:S208-S219. CrossRef Medline

Steinmann I, Gutschalk A (2012) Sustained BOLD and theta activity in auditory cortex are related to slow stimulus fluctuations rather than to pitch. J Neurophysiol 107:3458-3467. CrossRef Medline

Stevens CJ (2000) Acoustic phonetics. Cambridge, MA: MIT.

Talavage TM, Sereno MI, Melcher JR, Ledden PJ, Rosen BR, Dale AM (2004) Tonotopic organization in human auditory cortex revealed by progressions of frequency sensitivity. J Neurophysiol 91:1282-1296. Medline 
Terhardt E (1974) Pitch, consonance, and harmony. J Acoust Soc Am 55: 1061-1069. CrossRef Medline

Tierney A, Dick F, Deutsch D, Sereno M (2013) Speech versus song: multiple pitch-sensitive areas revealed by a naturally occurring musical illusion. Cereb Cortex 23:249-254. CrossRef Medline

Triantafyllou C, Hoge RD, Krueger G, Wiggins CJ, Potthast A, Wiggins GC, Wald LL (2005) Comparison of physiological noise at 1.5 T, 3 T, and $7 \mathrm{~T}$ and optimization of fMRI acquisition parameters. Neuroimage 26:243250. CrossRef Medline

Walker KM, Bizley JK, King AJ, Schnupp JW (2011) Multiplexed and robust representations of sound features in auditory cortex. J Neurosci 31: 14565-14576. CrossRef Medline
Wang X, Walker KM (2012) Neural mechanisms for the abstraction and use of pitch information in auditory cortex. J Neurosci 32:13339-13342. CrossRef Medline

Warren JD, Griffiths TD (2003) Distinct mechanisms for processing spatial sequences and pitch sequences in the human auditory brain. J Neurosci 23:5799-5804. Medline

Woods DL, Stecker GC, Rinne T, Herron TJ, Cate AD, Yund EW, Liao I, Kang X (2009) Functional maps of human auditory cortex: effects of acoustic features and attention. PLoS ONE 4:e5183. CrossRef Medline

Zatorre RJ, Belin P, Penhune VB (2002) Structure and function of auditory cortex: music and speech. Trends Cogn Sci 6:37-46. CrossRef Medline 